NBER WORKING PAPER SERIES

\title{
BEHAVIORAL HAZARD IN HEALTH INSURANCE
}

\author{
Katherine Baicker \\ Sendhil Mullainathan \\ Joshua Schwartzstein \\ Working Paper 18468 \\ http://www.nber.org/papers/w18468
NATIONAL BUREAU OF ECONOMIC RESEARCH
1050 Massachusetts Avenue
Cambridge, MA 02138
October 2012

We thank Dan Benjamin, David Cutler, John Friedman, Drew Fudenberg, Ben Handel, Ted O’Donoghue, Matthew Rabin, Jesse Shapiro, Andrei Shleifer, Jonathan Skinner, Douglas Staiger, Glen Weyl, Heidi Williams, and Danny Yagan for helpful comments. Schwartzstein thanks National Institute on Aging, Grant Number T32-AG000186 for financial support. The views expressed herein are those of the authors and do not necessarily reflect the views of the National Bureau of Economic Research.

At least one co-author has disclosed a financial relationship of potential relevance for this research. Further information is available online at http://www.nber.org/papers/w18468.ack

NBER working papers are circulated for discussion and comment purposes. They have not been peerreviewed or been subject to the review by the NBER Board of Directors that accompanies official NBER publications.

(C) 2012 by Katherine Baicker, Sendhil Mullainathan, and Joshua Schwartzstein. All rights reserved. Short sections of text, not to exceed two paragraphs, may be quoted without explicit permission provided that full credit, including (C) notice, is given to the source. 
Behavioral Hazard in Health Insurance

Katherine Baicker, Sendhil Mullainathan, and Joshua Schwartzstein

NBER Working Paper No. 18468

October 2012

JEL No. D01,D03,D8,I13

\begin{abstract}
$\underline{\text { ABSTRACT }}$
This paper develops a model of health insurance that incorporates behavioral biases. In the traditional model, people who are insured overuse low value medical care because of moral hazard. There is ample evidence, though, of a different inefficiency: people underuse high value medical care because they make mistakes. Such "behavioral hazard" changes the fundamental tradeoff between insurance and incentives. With only moral hazard, raising copays increases the efficiency of demand by ameliorating overuse. With the addition of behavioral hazard, raising copays may reduce efficiency by exaggerating underuse. This means that estimating the demand response is no longer enough for setting optimal copays; the health response needs to be considered as well. This provides a theoretical foundation for value-based insurance design: for some high value treatments, for example, copays should be zero (or even negative). Empirically, this reinterpretation of demand proves important, since high value care is often as elastic as low value care. For example, calibration using data from a field experiment suggests that omitting behavioral hazard leads to welfare estimates that can be both wrong in sign and off by an order of magnitude. Optimally designed insurance can thus increase health care efficiency as well as provide financial protection, suggesting the potential for market failure when private insurers are not fully incentivized to counteract behavioral biases.
\end{abstract}

Katherine Baicker

Professor of Health Economics

Department of Health Policy and Management

Harvard School of Public Health

677 Huntington Avenue

Boston, MA 02115

and NBER

kbaicker@hsph.harvard.edu

Sendhil Mullainathan

Department of Economics

Littauer M-18

Harvard University

Cambridge, MA 02138

mullain@fas.harvard.edu
Joshua Schwartzstein

Department of Economics

Dartmouth College

josh.schwartzstein@dartmouth.edu 


\section{Introduction}

Health insurance comes at a price: subsidizing health care causes people to use too much of it (Arrow 1963, Zeckhauser 1970, Cutler and Zeckhauser 2000). Moral hazard of this kind has been well documented empirically: there are many cases of people overusing expensive care that provides few health benefits (Newhouse 1993). At the same time, the "opposite" inefficiency is also well documented: the underuse of (often inexpensive) care that provides large health benefits. For example, glucose-lowering drugs increase life span, reduce the risk of limb loss or blindness and improve the quality of life for diabetic patients. Despite these benefits, many diabetics take these drugs sporadically, with only roughly $65 \%$ adherence (Rubin 2005). Diabetes is not the exception: the underuse of beta blockers for heart disease, medication to control HIV, antibiotics for tuberculosis, prenatal care by Medicaid populations (for whom it is free), and immunosuppresants after organ transplants are but a few other examples (van Dulmen et al. 2007). This inefficient underuse is hard to explain in the standard model. ${ }^{1}$ Parallel to moral hazard, we call the root (mis)behavior behavioral hazard: that is, misbehavior resulting from mistakes or behavioral biases, rather than privately optimal but socially suboptimal choices resulting from misaligned incentives. Under moral hazard, people overuse low value care because it is priced below cost. Under behavioral hazard, people fail to use high-value care or inefficiently use low-value care because of psychological biases. ${ }^{2}$ Factoring in behavioral hazard as well as moral hazard changes the fundamental trade-off between insurance and incentives.

Behavioral hazard does not come from one particular bias. Attention matters: choice of care may depend on the salience of symptoms, which is particularly problematic because many severe diseases have few salient symptoms. Timing matters: people may overweigh the immediate costs of care, such as co-pays and hassle-costs of setting up appointments or filling prescriptions. Memory matters: people may simply forget to take their medications or refill their prescriptions. Beliefs matter: people may have false beliefs and poor learning mechanisms about the efficacy of different

\footnotetext{
${ }^{1}$ In principle, it is possible to argue that unobserved costs of care, such as side effects, drive what seems to be underuse. However, in practice, this argument is difficult to make for many of the examples we review. It is also worth noting that the underuse we focus on is very different from the underuse that can arise in dynamic moral hazard models. In such models, patients may underuse preventive care that generates future monetary costs for the insurer (Goldman and Philipson 2007). Here, we focus on the underuse of care whose benefits outweigh costs to the consumer. For example, though the underuse of diabetes medications does generate future health care costs, the uninsurable private costs to the consumer alone (e.g. higher mortality and blindness) make non-adherence likely to be a bad choice even if she is fully insured against future health care costs. We also abstract from underuse due to health externalities (e.g. the effect of vaccination on the spread of disease).

${ }^{2}$ While our examples often focus on underuse, behavioral hazard can also produce overuse of care. For example, people seek out antibiotics for children's ear infections, an often ineffective treatment, even at prices near true costs (Spiro et al. 2006). Older men demand surgery following a diagnosis of prostate cancer when "watchful waiting" would be more effective (for patients themselves) and lower cost (Cohen et al. 2008; Liebman and Zeckhauser 2008). Overuse of MRIs for low back pain doubles the number of surgeries with no improvement in outcomes (Jarvik, 2003).
} 
treatments. Differentiating between biases is clearly important in designing targeted behaviorallyinformed interventions, but we show that significant progress can be made in analyzing the effects of traditional price levers without such differentiation. Following Mullainathan, Schwartzstein and Congdon 2012, we nest a broad set of behavioral biases in a single model of behavioral hazard that yields welfare calculations, optimal copay formulas, and market equilibrium predictions that are functions of reduced-form elasticities and do not depend on the specific psychological primitives. ${ }^{3}$ 4

The presence of these psychological factors changes the central insights of the standard model. Consider the welfare impact of reducing copays. Typically this effect is decomposed into two parts: an insurance value and a moral hazard cost. A key feature is that the moral hazard cost can be inferred directly (and solely) from the demand response. The consumers who respond to reductions in the copay are the ones who are close to indifference. Since copays are below cost, this means the marginal consumers are inefficiently using care: they must value it less than it truly costs. This insight is used to calculate the deadweight cost due to moral hazard (which is weighed against some measure of insurance value which is calculated separately). For example, the RAND Health Insurance Experiment suggests a demand elasticity of roughly .2 by comparing demand under a plan with no coinsurance to demand under one with 95 percent coinsurance (Manning et al. 1987, Feldman and Dowd 1991). This implies that the moral hazard induced by moving from the plan with $95 \%$ coinsurance to the one with no coinsurance generated a welfare loss of \$291 per person from moral hazard (in 1984 dollars). Importantly for empirical work, this inefficiency can be inferred without analyzing the impact on actual health, since the health benefit equals the copay on the margin. ${ }^{5}$

Behavioral hazard alters this logic. When consumers are biased, the marginal consumer may not have a true marginal health benefit equal to the copay. The rise in demand may not come from a consumer who is inefficiently using more care. Instead it might come from one who was underusing the treatment before. This means that lowering copays could increase welfare by alleviating behavioral hazard rather than reduce welfare by worsening moral hazard. We cannot tell which case we are in from the demand response alone. Empirical work suggests that this is more than an

\footnotetext{
${ }^{3}$ Chetty (2009) proposes a model of salience and taxation in a similar way: he derives empirically implementable formulas for the incidence and efficiency costs of taxation that are robust across positive theories for why agents may fail to incorporate taxes into choice.

${ }^{4}$ We take the policy-maker's welfare function as given. This sidesteps a challenge that every exercise in behavioral welfare economics faces. How should the policy-maker reconcile in a systematic way individuals' inconsistent preferences? See Bernheim and Rangel (2009) for a discussion of a choice-based approach to recovering consistent welfare functions from inconsistent choice behavior. In effect, we are assuming in Bernheim and Rangel's framework that there is some (unmodeled in our framework) ancillary condition that the policy-maker uses to infer a consistent preference for the agent and the objective of the welfare problem is to maximize this preference.

${ }^{5} \mathrm{We}$ can only equate the marginal private utility benefit with the co-pay, but presumably much of this benefit derives from the health effects.
} 
abstract concern: higher copays seem to dissuade the use of high-value care as much as of lowvalue care, suggesting that behavioral hazard is quite prevalent. Our model provides guidance in some cases: it shows that the welfare impact of copay changes can be calculated using both demand and health responses, which is useful when we can confidently bound the health responses relative to unobserved costs or benefits of care. We apply this approach to a large scale field experiment that eliminated copays for recent heart attack victims (Choudhry et al. 2011). Taking behavioral hazard into account proves quantitatively important: the standard moral hazard formula implies that eliminating copays generated a modest marginal welfare cost; but once behavioral hazard is taken into account this becomes a marginal welfare gain that is ten times larger.

In the same way, behavioral hazard changes the optimal design of insurance. In the standard model, the optimal copay increases in the elasticity of demand, since it must balance this measure of moral hazard against insurance value (Zeckhauser 1970). With behavioral hazard, in contrast, the optimal copay formula now depends on both demand and health responses. This formula provides a formal foundation for "value-based insurance design" proposals that argue for lower cost sharing for higher value care (Chernew, Rosen, and Fendrick 2007); our model nests a more specific result of Pauly and Blavin (2008) that applies to the case of uninformed consumers. ${ }^{6}$ For example, it implies that we should optimally have zero copays for drugs where (marginal) health benefits are large and demand is elastic. ${ }^{7}$ The introduction of behavioral hazard thus does not just suggest novel "psychological" interventions or "nudges" (Thaler and Sunstein 2009), but fundamentally changes how we analyze standard price levers. Nevertheless, our framework also allows us to analyze the use of nudges, such as defaults and reminders, to mitigate overuse and underuse. We show that responses to nudges can also be used to calculate the welfare impact of policy changes in the absence of health data: the extent of behavioral hazard can be inferred from comparing the demand response to prices to the demand response to nudges.

Finally, behavioral hazard changes our understanding of the structure of health insurance markets. Insurance no longer provides only financial protection: it can also increase the efficiency of health care utilization by reducing behavioral hazard (rather than just reducing efficiency by creating moral hazard). This may shed light on recent evidence of the health impact of expanding insurance coverage (Finkelstein et al. 2011, Baicker and Finkelstein 2011). It also implies a novel insurance market failure: even though optimal insurance could reduce behavioral hazard, market-provided insurance may not. If enrollees were sophisticated about their behavioral biases perfectly predicting their behavioral hazard and insuring accordingly - then market-provided insurance would be optimally designed to counteract the biases. ${ }^{8}$ But with naive enrollees, insurers have

\footnotetext{
${ }^{6}$ Liebman and Zeckhauser (2008) make a similar point, though they do not provide a formal model.

${ }^{7}$ We may even want negative copays when possible, e.g., paying people to take highly effective medications.

${ }^{8} \mathrm{We}$ ignore ex ante heterogeneity among consumers and issues of selection. Of course, adverse selection provides a rationale for government intervention even in the standard model.
} 
less incentive to mitigate underuse since naive consumers will not fully value co-pays designed to counteract their biases. ${ }^{9}$ These problems are especially severe when insurers have nudges as well as prices available and when they have limited time horizons (and thus do not bear the full cost of enrollees' future health care use). We show that this accords with some existing patterns of insurance plan features and coverage.

The paper proceeds as follows. Section 2 makes the case for the need to incorporate behavioral hazard into the standard model, laying out evidence of behavior inconsistent with moral hazard alone. Section 3 presents the model, beginning with traditional moral hazard and then adding behavioral hazard. Section 4 considers optimal design and Section 5 develops tools for empirically calibrating the welfare impact of co-pay changes as well as the optimal co-pay formula. Section 6 analyzes the equilibrium insurance contract and examines when and how this contract differs from the optimum. Section 7 concludes. Proofs are in the Appendix, as are some extensions of the basic model and a case study applying the model to treatment of hypertension.

\section{Behavioral Hazard}

\subsection{Evidence}

In this section, we describe some evidence that is meant to motivate our analysis of behavioral hazard. Table 1a summarizes some examples suggestive of underuse. Much of the evidence on underutilization comes from (lack of) adherence to prescribed drugs. Partly this is because costs (including side effects) and benefits are often better measured for drugs than for other treatments. In the case of diabetes, for example, estimates suggest that despite the dire health consequence of unmanaged diabetes, adherence to glucose-controlling drugs is only between 60 and $80 \%$ (Rubin 2005). One study showed that almost half of diabetic patients did not have their prescriptions filled consistently, despite the fact that consistent filling cut the risk of hospitalization in half (Sokol et al., 2005). Adherence appears to be even lower among Medicaid recipients, for whom the cost of the medication is zero. Underuse is of course not restricted to prescription drug non-adherence. ${ }^{10}$ Patients do not receive recommended care across a wide range of categories, with only 55 percent receiving recommended preventive care including screenings (e.g., colonoscopies) and followup care for conditions ranging from diabetes and asthma management to post-hip-fracture care

\footnotetext{
${ }^{9}$ Insurers will face some incentive to counteract negative behavioral hazard since naive consumers over-estimate their demand in this case, which creates some benefit to reducing copays. But negative behavioral hazard will not be efficiently managed in equilibirum.

${ }^{10}$ Cleemput et al. (2002) note some of the methodological challenges in cost-benefit analysis of non-compliance.
} 
(McGlynn et al., 2003; Denberg et al., 2005; Ness, 2000). ${ }^{11} 12$

While highly suggestive of behavioral factors at work, this evidence can nevertheless be interpreted through the lens of the standard model. Health care can have unobserved costs (such as side-effects) or unobserved benefits (such as hard-to-quantify reductions in pain). There can be heterogeneous treatment effects and patients might know (or learn) that certain treatments do not work for them despite high average returns or do work for them despite low average returns. As a result, choices that appear to reflect some bias might actually be optimal. These arguments are surely relevant in some cases. For example, reducing obesity through exercise and calorie control decreases mortality and morbidity. Yet many overweight people over-eat and fail to exercise. Labeling these behaviors as suboptimal or irrational may reflect misinterpretation based on the mismeasurement of the private costs of exercise and the private enjoyment from food. Our research has definitively determined that running is unpleasant and donuts are tasty! ${ }^{13}$

Nonetheless, as we argue in more detail after presenting the formal model, some of the behaviors described in Table 1a likely reflect misutilization due to behavioral hazard. First, the magnitudes involved often make the case for unobservable factors less plausible, as discussed in greater detail in Section 5. It is difficult to argue that preferences are consistent with rationally choosing not to pay a $\$ 5$ copayment and swallowing a pill with minimal side-effects when it produces a substantial reduction in the probability of mortality. To take one particularly striking example, relatively small reductions in copayments even after an event as salient as a heart attack still produce improvements in adherence: providing post-heart attack medications for free (instead of roughly a $\$ 10-\$ 25$ copayment) increased adherence by about 5 percentage points (relative to a base of 35-50 percent), and this increase was associated with a reduced rate of subsequent major vascular events (Choudhry et al., 2011). Second, while there is plenty of evidence of heteroge-

\footnotetext{
${ }^{11}$ For example, less than 66 percent of recommended immunizations were delivered, 54 percent of recommended care for asthma patients, and 45 percent of recommended care for diabetics. Rates of recommended screening for colorectal cancer are only $40 \%$, despite the fact that one-time screening among younger populations is highly cost-effective and estimates suggest compliance would prevent almost 10,000 deaths annually (Denberg et al., 2005; Ness, 2000). Prenatal care has an important effect on the health and survival of newborns, but despite expansions of Medicaid to many low-income pregnant women, more than 30 percent of women do not receive prenatal care in the first trimester of pregnancy (Currie and Gruber 1996; Institute of Medicine; HRSA). An NCQA study (2005) concluded that half of chronically ill patients are not compliant with recommended care.

${ }^{12}$ Evidence on the effects of expanding health insurance is consistent with these patterns of underuse. For example, in a recent randomized trial expanding public health insurance to low-income adults, insurance coverage increased use of preventive care such as mammograms by $60 \%$, blood sugar testing for diabetes by $15 \%$, cholesterol monitoring by $20 \%$, and pap smears by $45 \%$ (Finkelstein et al., 2011; Baicker and Finkelstein 2011). While we would expect insurance to increase use (because of both subsidized copays and relaxation of budget constraints with imperfect credit markets), the magnitude of the change in use of care with high marginal value and moderate costs seems likely to be driven by behavioral factors as well.

${ }^{13}$ However, there is some evidence suggesting that even for these examples there are behavioral forces at work. Individuals display signs of time inconsistency in both of these cases, reporting a desire to change behavior but not doing so.
} 
neous treatment benefits, there is less indication that people self sort in the manner predicted by the standard moral hazard model. For example, Goldman et al. (2006) estimate that a \$10 increase in copayments drives similar reductions in use of cholesterol-lowering medications among those with high risk (and thus high health benefits) as those with much lower risk. Finally, observed reactions to "nudges" further builds the case for behavioral hazard. If people are simply rationally trading off current costs and future benefits, then a text message reminder or simplification of the dosage schedule should not produce a substantial change in adherence, but there is ample evidence that such nudges do produce substantial changes (Schedlbauer et al. 2010; Schroeder et al. 2004; Strandbygaard et al., 2010). ${ }^{14}$

It is also worth noting that there are examples of overuse that are hard to reconcile with the standard model, as summarized in Table $1 \mathrm{~b} .{ }^{15}$ The case of (over)treatment of back pain is illustrative. Back pain is wide-spread and expensive: it is the second leading symptomatic cause of physician visits and cost more than \$25 billion in 1998 (Deyo 1992, 2006; Strine 2007, Luo 2004). Routinely doctors prescribe fMRIs despite evidence that suggests they are ineffective (Di Iorio, 2000, Jarvik, 2003; American College of Radiology 2010; Chou 2009; Sheehan 2010). Similarly, there is also mounting evidence of the overuse of detection and treatment of prostate cancer. Because the disease is rarely fatal and the cancers are quite slow-growing, the five-year survival rate for those diagnosed is 99.4\% (Howlader et al., 2011). Perlroth, Goldman, and Garber (2010) show that "watchful waiting" is as effective as more expensive clinical teatments, yet $58 \%$ of prostate cancer patients pursue these more aggressive options - costing Medicare \$1.7-\$3.0 billion, exposing patients to risk, and not improving their prognosis. ${ }^{16}$ Unlike the evidence on underuse, though, it is practically difficult to rule out psychic benefits that drive this overuse (such as "peace of mind"), making it difficult to empirically separate overuse due to behavioral hazard from that due to moral hazard. While our model allows for overuse that is behaviorally motivated, most of the examples we focus on are thus of underuse.

\footnotetext{
${ }^{14}$ For example, adherence to statins is far from perfect and is responsive to nudges and small copayment changes. Schedlbauer et al. (2010) note that poor patient adherence "is a major factor in the lack of success in treating hyperlipidaemia." They find that nudges focusing on reminders and reinforcements were particularly promising, with four out of six trials reviewed producing significant increases in adherence ranging from 6-24 percentage points. Similarly, simplifying the dosing schedules for blood pressure medication can lead to a 10-20\% increase in adherence (Schroeder et al., 2004) and text reminders increased adherence to asthma medication by almost 20\% (Strandbygaard et al., 2010).

${ }^{15}$ Of course, there are many examples of overuse consistent with traditional moral hazard - where patients face very low marginal costs and consume care with extremely high social costs but not commensurately high health benefits. For example, one study of the use of Cetuximab (Erbitux) for non-small cell lung cancer calculated a total cost of $\$ 80,352$, but only increased overall survival by 1.2 months - or a cost of $\$ 800,000$ per life-year (Fojo et al. 2009).

${ }^{16}$ Testing for prostate cancer may be similarly overused. Prostate specific antigen (PSA) testing has been performed on most men in the US over age 50 (Ross, Berkowitz, \& Ekwueme, 2008). However, recent large scale clinical trials indicate that PSA screening has, at best, a small effect in reducing mortality, and that the risks likely outweigh the benefits (Andriole et al. 2009, Schröder et al. 2009, M. Djulbegovic et al., 2010).
} 


\subsection{Underlying Psychologies}

What drives behavioral hazard? The literature has uncovered several different psychological forces.

Individuals appear to overweight salient symptoms and underweight less salient ones (Osterberg and Blaschke 2005). Salience of symptoms relative to the costs and benefits of care can drive overuse or underuse. In the case of diabetes, symptoms of elevated glucose levels are not salient (Rubin 2005), and it is easy to undervalue the health benefits of taking a pill whose effects cumulate slowly over time. ${ }^{17}$ Rates of recommended screening for colo-rectal cancer are only $40 \%$ (compared with around $75 \%$ for mammograms), despite the fact that one-time screening among younger populations is highly cost-effective (Denberg et al., 2005; Ness, 2000), perhaps because there are no salient symptoms that would drive one to get screened. Patients at stage B or C of HIV/AIDS (symptomatic stages) are more likely to be adherent to their treatment regimens than are patients at stage A (the asymptomatic stage) (Gao et al. 2000). With tuberculosis, most treatment regimins are at least six months in length, but effective therapy leads to improved symptoms after the first four weeks and a concurrent drop-off in adherence. Similarly, salience may also drive overuse. Pain, for example, is highly salient. Even knowing in the abstract that the pain may selfresolve, patients may overweight the current pain and push for costly tests and treatments. Stories in the popular press highlight the role of symptom salience: a recent report noted the death of an uninsured patient with a tooth infection who was prescribed an antibiotic and a painkiller and who spent his limited resources to fill the painkiller prescription (ABC news, 2011).

Other biases are likely important as well. Present bias (Laibson 1997, O'Donoghue and Rabin 1999) may drive behavior when the benefits of care are in the distant future but its costs appear in the present. In these cases, present bias would lead one to under-use care. For example, the hassle of obtaining prenatal care is paid now, whereas the benefits happen later. False beliefs could play a role. Tuberculosis patients may stop taking their antibiotics halfway through their drug regimen not just because salient symptoms have abated but also because they believe the disease has disappeared. People may falsely attribute efficacy as well. For example, people may confuse reversion to the mean with treatment efficacy.

Finally, people may simply forget. In addition to forgetting to take medication on a given day, people can forget to refill prescriptions. A related phenomenon is "white coat adherence", whereby patients tend to improve their adherence 5 days before and after a doctor's appointment compared to 30 days after (Osterberg and Blaschke 2005). Who among us does not floss more regularly right before a dentist appointment?

Given this evidence, how should insurance contracts optimally respond to the presence of behavioral hazard?

\footnotetext{
${ }^{17}$ There are short term benefits of controlling glucose, such as increased energy and better quality sleep, but many patients do not recognize these benefits (Rubin 2005).
} 


\section{Model Incorporating Moral Hazard and Behavioral Hazard}

We begin with a stylized model of health insurance. Consider an individual with wealth $y$. Insurance has price, or premium, $P$. When healthy the individual has utility $U(y-P)$ if she buys insurance. With probability $q$, she can fall sick with a varying degree of severity $s$ that is private information on the part of the individual. For example, individuals may be afflicted with asthma that varies in how much it debilitates. In Appendix B, we extend the model to consider a situation with multiple illnesses and treatments covered by the same insurance contract. Assume $s \sim F(s)$, where $F$ has support on $\mathcal{S}=[\underline{s}, \bar{s}] \subset \mathbb{R}^{+}$and $\underline{s}<\bar{s}$. Assume further that $F(s)$ has strictly positive density $f(s)$ on $\mathcal{S}$. Severity is measured in monetary terms so that when sick, absent treatment, the agent receives utility $U(y-P-s)$.

Treatment however can lessen the impact of the disease. Treatment costs $c$ and its benefit $b(s)$ depends on severity. The more severe the disease the greater the benefits: $b^{\prime}(s)>0$. We also assume $b(0)=0$ (the unaffected get no benefit) and $b^{\prime}(s) \leq 1$ (the treatment cannot at the margin make the person better off than not having the disease). Once again the benefits are put in monetary terms. It is efficient for some but not all of the sick to get treated: $b(\underline{s})<c<b(\bar{s})$. We assume that the insured individual pays price or "co-pay" $p$ to receive treatment. Notice that the price $p$ is independent of severity; we implicitly assume that severity of any given disease cannot be contracted over because the insurer cannot perfectly measure it. The interpretation is that the co-pay is conditional on all information known to the insurer (e.g., the disease and treatment), but the individual may have some residual private information. In this way, we nest the traditional moral hazard model. An insured individual who receives treatment for his disease gets utility $U(y-P-s+b(s)-p)$.

\subsection{Moral Hazard}

In this simple setup the choice to receive treatment when insured is easy: the rational person gets treated whenever benefits exceed price: $p<b$. This decision is the source of moral hazard. As we will see, the insurance value in insurance comes from setting price below true cost. This subsidized price can generate distortions. Individuals should efficiently get treated whenever $b>c$, but they get treated whenever $b>p$. So individuals for whom benefits are in between the price and the cost $p<b<c$ are inefficiently receiving service: privately, getting treated makes sense (benefits exceed private costs) but socially it does not (benefits do not exceed social cost). Figure 1 illustrates this issue of moral hazard that is at the heart of the insurance design problem. Individuals are arrayed on the line according to treatment benefits. Those to the right of the cost $c$ should receive treatment and do. Those to the left of the price $p$ should not receive treatment and do not. The middle region represents the problem: those individuals should not receive treatment but they do. Raising the 
price individuals face diminishes this over-utilization, but as we will see this comes at the cost of diminished insurance value.

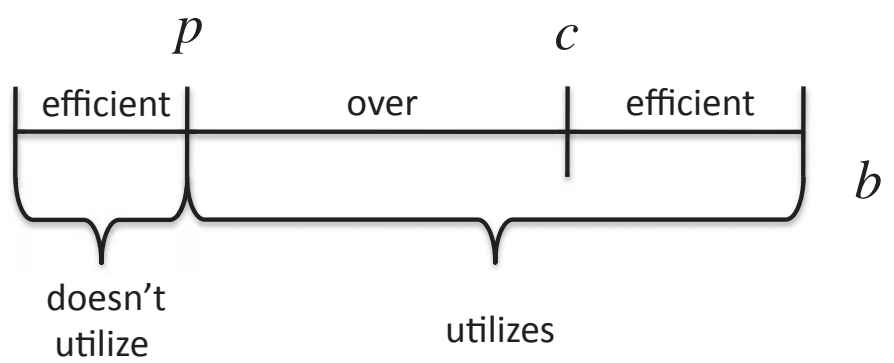

Figure 1: Standard Model

Notice a key prediction of the standard model: insurance is the source of misutilization. Since people accurately trade off the benefits and costs of medical care, misutilization stems from copays deviating from social cost. More formally, define $m(p)$ to be an individual's demand for care at a given price: for the rational agent, it equals 1 if and only if $b(s)>p$. Now let

$$
H(p)=E[m(p) b-s]
$$

represent the aggregate level of health and define

$$
\mathcal{E}(p)=H(p)-c M(p)
$$

as the level of health net of the costs of supplying that level, where $M(p)=E[m(p)]$ is the aggregate demand for medical care at a given copay level $p . H(p)$ represents the expected value of disease severity post treatment decisions at co-pay level $p$ (again, in income-equivalent units), and $\mathcal{E}(p)$ nets out the costs of treatment at that co-pay level. An efficient level of health is generated when $H^{\prime}(p)=c M^{\prime}(p)$, which holds if and only if $p=c$. That is, the amount of care consumed is efficient if and only if people are uninsured and bear the full costs of care. When $p<c$, the resources devoted to producing health are inefficiently high. ${ }^{18}$

\footnotetext{
${ }^{18}$ For simplicity, we are assuming away income effects or issues of affordability. In a standard framework, insurance could lead to more efficient levels of health insofar as it makes high-value, high-cost procedures affordable to consumers (Nyman 1999). However, in this framework, insurance cannot lead consumers to make more efficient decisions on the margin. Abstracting from income effects serves to highlight this well-known fact (Zeckhauser 1970).
} 


\subsection{Incorporating Behavioral Hazard}

As the discussion in Section 2 suggests, however, there is ample evidence of behavior that is diffi-

cult to interpret as a rational actor's response to subsidized prices. We next incorporate behavioral hazard alongside the traditional moral hazard.

\subsubsection{Capturing Diverse Psychologies in One Framework}

We incorporate behavioral hazard through a simple modification of the original model. Instead of decisions being driven by a comparison of true benefits to price, evaluating whether $b(s)>p$, people choose according to whether $b(s)+\varepsilon(s)>p$, where $\varepsilon$ is positive in the case of positive behavioral hazard (for example, seeking an ineffective treatment for back pain) and negative in the case of negative behavioral hazard (for example, not adhering to effective diabetes treatment). We assume that $b(s)+\varepsilon(s)$ is differentiable and strictly increasing in $s$.

When behavioral biases exist, other non-price levers - "nudges" - also affect demand. For example, as detailed in Section 2, reminders or explanations that make the costs of non-adherence more salient can affect utilization, as can hassle factors like making it difficult to make a doctor's appointment or requiring prior authorization to refill a prescription. Formally, there is a set $\mathcal{N}$ of nudges available to insurers, where a nudge is modeled as affecting demand through influencing the behavioral error $\varepsilon$. The direct cost to the insurer of nudge $n$ is $\psi(n) \geq 0$. Suppose there is a "default nudge" $n=0 \in \mathcal{N}$ with $\psi(0)=0$. Unless otherwise noted, we assume the insurer sets the default nudge and notationally suppress the relationship between the nudge and the behavioral error.

This formulation follows Mullainathan, Schwartzstein and Congdon (2012) and implicitly captures a divide between preference as revealed by choice and utility as it is experienced, or between "decision utility" and "experienced utility" (Kahneman et al. 1997). In our framework $b-p$ affects the experienced utility of taking the action. Individuals instead choose $a s$ if $b+\varepsilon-p$ affects this utility. We focus on behavioral models that imply a clear wedge between these two objects - in other words, models that generate a non-zero $\varepsilon$ term, or an "internality". This clarifies a category of behavioral models that lies outside our focus: models of non-standard preferences. For example, anticipation and anxiety may alter how individuals experience benefits (Koszegi 2003): benefits will vary depending on whether taking the action (such as getting an HIV test) leads to anxiety in anticipating the outcome. In these kinds of situations it may be wrong to model the behavioral factor as a bias affecting $\varepsilon$, but rather as a force that affects the mapping between outcomes (such as getting a diagnostic test) and benefits $b$.

Note also a simplifying feature of the basic model: biases are homogeneous and expressed with certainty, conditional on the disease and severity - i.e., $\varepsilon(s)$ is non-stochastic (fixing $s$ ). The case 
where $\varepsilon$ is stochastic is formally considered in Appendix B, where this extension allows us to analyze psychologies like forgetfulness, where $\varepsilon(s)$ is strongly negative only with some probability. ${ }^{19}$ Allowing for heterogeneity in the degree to which people are biased does not substantively affect the normative analysis, but complicates the presentation.

At the same time, this simple formalization captures a variety of behavioral phenomena. Three examples are summarized here.

Example 3.1. Present-bias (e.g., Laibson 1997, O'Donoghue and Rabin 1999)

Take the canonical $(\beta, \delta)$ model of present-bias, where, for simplicity, $\delta=1$. Suppose each treatment is associated with an immediate cost but a delayed benefit. Formally, $b(s)=v(s)-$ $k(s)$, where $v(s)$ represents delayed benefits and $k(s)$ represents immediate costs. While standard agents (for simplicity) are assumed not to discount future benefits, behavioral agents discount these benefits by factor $\beta \in(0,1)$, quantifying the degree to which they are present-biased. Instead of getting treated whenever $b(s)=v(s)-k(s)>p$, present-biased agents get treated whenever $b(s)+\varepsilon(s)=\beta v(s)-k(s)>p$. In this case, $\varepsilon(s)=-(1-\beta) v(s)$. Given this micro-foundation, behavioral hazard is negative whenever the benefits of treatment are delayed, where the magnitude of the bias is increasing in these benefits. (Behavioral hazard is positive if benefits are immediate and costs are delayed, corresponding to the situation where $k(s)<0, v(s)<0$.)

Example 3.2. Inattention (e.g., DellaVigna 2009)

Consider DellaVigna's (2009) model of limited attention and suppose the severity of symptoms is the sum of three components: the severity of highly visible or painful symptoms, $v$, the severity of opaque or non-painful symptoms, $n$, and other symptoms, $o$. Formally,

$$
s=v+n+o .
$$

The behavioral agent overweighs the painful symptoms and underweighs non-painful symptoms, so he acts not on true disease severity $s$, but on "decision severity"

$$
\hat{s}=\alpha v+\beta n+o,
$$

where $\alpha>1$ and $\beta<1$. Thus,

$$
\begin{array}{r}
b(s)+\varepsilon(s)=b(v+n+o)+\varepsilon(s)=b(\alpha v+\beta n+o) \Rightarrow \\
\varepsilon(s)=b(\alpha v+\beta n+o)-b(v+n+o) .
\end{array}
$$

\footnotetext{
${ }^{19}$ Allowing for forgetfulness does not change how copays affect welfare, but the equilibrium co-pay does depend on the level of forgetfulness since it influences the degree to which the agent misforecasts her demand for treatment.
} 
From (5) we see that behavioral hazard is negative whenever non-painful symptoms are sufficiently prominent (i.e., $n>v(\alpha-1) /(1-\beta)$ ) and is positive whenever painful symptoms are sufficiently prominent (i.e., $v>n(1-\beta) /(\alpha-1)$ ). The magnitude of the hazard depends on the composition of painful and non-painful symptoms.

\section{Example 3.3. False beliefs}

An extremely simple example consistent with our formulation is one where agents mis-estimate or have false beliefs about the benefit to getting treated. For example, people may overweigh the benefits to buying an herbal medicine with no known efficacy. Instead of getting treated when $b(s)>p$, such agents get treated when $\hat{b}(s)>p$, where $\hat{b}(s)$ is the decision benefit to getting treated. In this case, $\varepsilon(s)=\hat{b}(s)-b(s)$. Under this model, behavioral hazard is positive whenever agents overweigh the benefits of treatment $(\hat{b}(s)>b(s))$ and is negative whenever agents underweigh the benefits of treatment $(\hat{b}(s)<b(s))$.

\subsubsection{Implications for the Demand Curve}

No matter the micro-foundation, behavioral hazard changes how we think about the demand for treatment. We illustrate this in Figure 2. We have now added a second axis to form a square instead of an interval. The vertical axis now represents $b+\varepsilon$, once again recognizing that $b+\varepsilon$ can vary by individual. The horizontal line now separates the region where $b+\varepsilon>p$. We see the ranges of misutilization are no longer clear. The people in the bottom left corner (where $b+\varepsilon<p$ and $b<c$ ) are efficient non-users. Those in the top right corner (where $b+\varepsilon>p$ and $b>c$ ) are efficient users. But there are now three other interesting regions.

The bottom right area is a region of underutilization. People fail to consume care in this region because $b+\varepsilon<p$ but the actual benefits exceed social cost. Such a region is intriguing because the traditional model does not generate underutilization. Examples from Table 1a illustrate such underutilization. The top left area illustrates overutilization. In this area, benefits of care are below cost so $b<c$, and the efficient outcome is for the individual not to get treated. Yet because $b+\varepsilon>p$ the behavioral agent receives care. This area can be broken down further, according to whether $b+\varepsilon>c$. When this inequality holds, decision benefits are above cost even though true benefits are below cost. In this case, overutilization will not be solved by setting price at true cost. Examples from Table 1b illustrate such overutilization.

In short, misutilization is not solely a consequence of health insurance in the behavioral framework. Insurance can thus lead to more efficient health delivery by counteracting biases. In particular, health net of costs, $\mathcal{E}(p)$, is maximized not at $p=c$ but rather when $p=c+\varepsilon$, where $\varepsilon$ should be evaluated at the disease severity of the marginal agent given co-pay $p$. The efficient level 


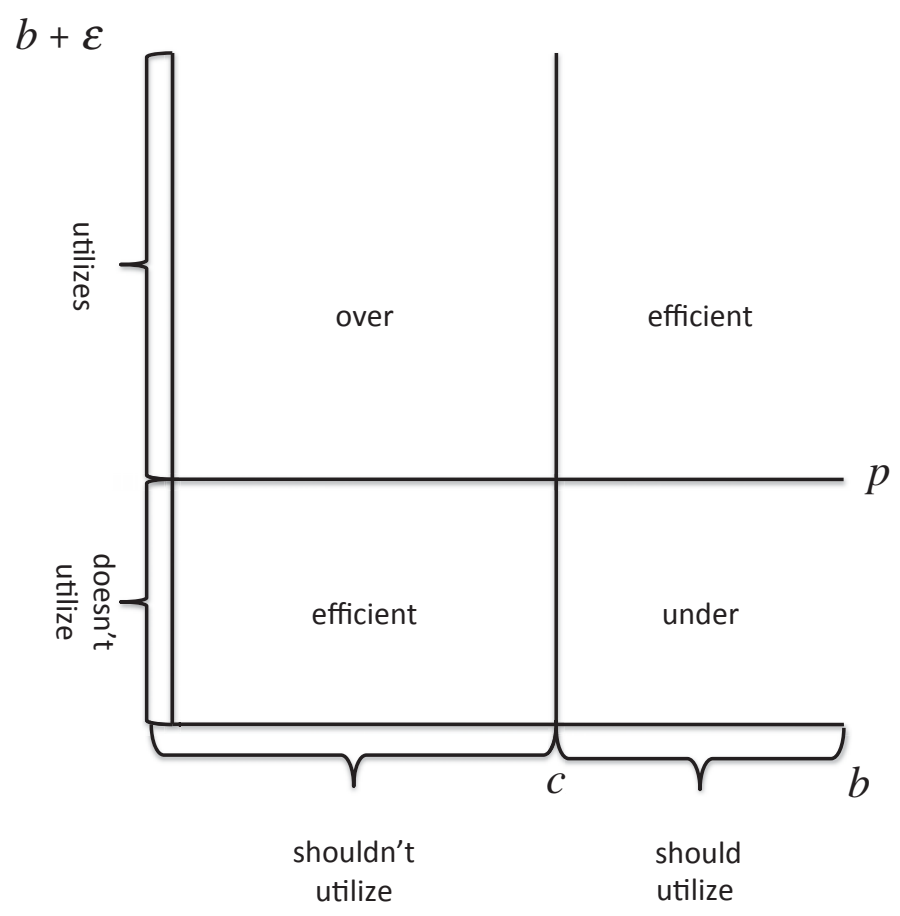

Figure 2: Model with Behavioral Hazard

of health may be greater or lower than that absent insurance. Given that health insurance can have this benefit, how is it efficiently designed?

\section{Optimal Health Insurance Design}

Using this more flexible model, we first solve for the socially optimal insurance policy (before turning to what the market is likely to provide). To begin, we model an insurance policy as having only two components: the fixed premium $P$ paid irrespective of care received and the price paid for care $p$ that cannot be based on $s$. (Later, we will consider what happens when insurers can also set nudges.) Since the baseline model only considers a single disease and treatment, the co-pay can be interpreted as conditional on all information known the insurer, e.g., the diagnosis and treatment. ${ }^{20}$ Recall that $m(p)$ represents an individual's demand for care at a given price: it equals 1 if and only if $b(s)>p$ for the rational agent and $b(s)+\varepsilon(s)>p$ for the behavioral agent.

We focus first on a benevolent social planner designing health insurance to maximize social

\footnotetext{
${ }^{20}$ Appendix B considers a situation with multiple illnesses and treatments exogeneously covered by the same co-pay.
} 
welfare. Specifically, it maximizes:

$$
W=(1-q) U(y-P)+q E[U(y-P-s+m(p)(b-p)) \mid \text { sick }] .
$$

$W$ represents the expected utility. The first term is the utility if individuals do not get sick: they simply pay the premium. The second term is the utility if they do get sick: the expected utility (depending on disease severity) which includes the loss due to being sick $(-s)$ as well as the benefits of care net of costs to individuals $(b-p)$ for the times they choose to use care $(m(p)=1)$. We assume that the social planner faces a break-even constraint - insurance must be self-funding: $P=M(p)(c-p)$.

To understand how the social planner should design insurance, it is useful to think about how social welfare changes as the co-pay $p$ changes. Assume the (positive) copay is below cost $c$ and we are considering an increase. Absent a demand response, a rise in co-pay lowers utility for people who are sick enough that they demand treatment. Since these are the times when individuals have the highest marginal utility (by assumption), this produces a negative effect of increasing the copay: it diminishes insurance value. ${ }^{21}$ Of course, people's choice of care could change. We will begin by focusing on rational actors.

\subsection{Rational Actors}

Consider in Figure 3 the effect of raising the price from $p<c$ to $p^{\prime}$. All those whose benefit of treatment falls between $p$ and $p^{\prime}$ will now no longer consume that care. Assuming this change is small, $p^{\prime}<c$, so benefits for these marginal individuals lie below costs and increasing the co-pay has the benefit of reducing overutilization. This produces the basic tradeoff of insurance design when people are rational: a higher price reduces moral hazard but lowers insurance value.

This intuitive trade-off has a quantitative analogue. Differentiating $W$ with respect to $p$ subject to the break-even constraint, and converting into a money metric by normalizing the increase in welfare by the welfare gain from increasing income by 1 yields: ${ }^{22}$

$$
\frac{d W}{d p} / \frac{d W}{d y}=-M^{\prime}(p)(c-p)-I \cdot M(p),
$$

\footnotetext{
${ }^{21}$ To be more precise, our assumptions guarantee that a rise in the co-pay from $p \in(0, c)$ diminishes insurance value when all individuals are rational actors because, in that case, the sick who demand treatment are worse off than the sick who do not, even post treatment. Since this may not hold with behavioral hazard, stronger conditions (for example, that $q$ is sufficiently small) are necessary to guarantee that the people who demand treatment on average have higher marginal utility than those who do not.

${ }^{22}$ Demand $M(p)$ is differentiable almost everywhere, but may not be differentiable at $p^{\min }=\inf \{p: M(p)<q\}$ or $p^{\max }=\sup \{p: M(p)>0\}$. In both these cases, $M(p)$ still has left- and right-hand derivatives. To ease notation, take $M^{\prime}(p)$ to be the right-hand derivative unless otherwise noted, with the understanding that this equals the derivative almost everywhere.
} 


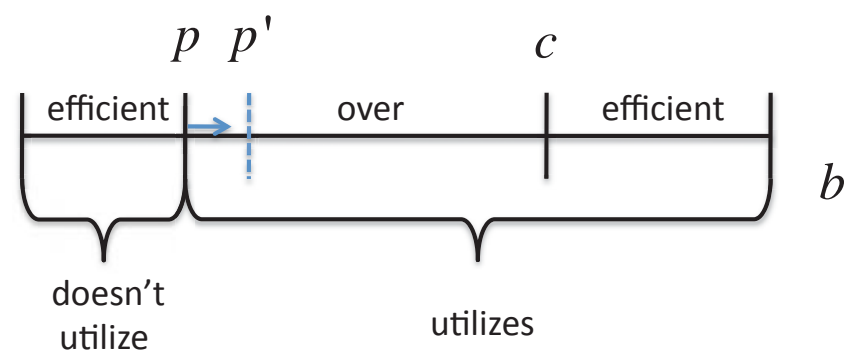

Figure 3: Effect of Changing the Co-pay on Utilization in the Standard Model

where $I=\left(\frac{E\left[U^{\prime}(C) \mid m=1\right]-E\left[U^{\prime}(C)\right]}{E\left[U^{\prime}(C)\right]}\right)$ equals the insurance value to consumers $(C=y-P-s+$ $m(b-p))^{23}$

Equation (7) captures the trade-off discussed above. The first term, $-M^{\prime}(p)(c-p)$, represents the welfare gain from reducing moral hazard: it can be thought of as the number of people who are at the margin multiplied by the difference between the social cost and social value of their treatment - the marginal inefficiency $-(c-p)$. Note that the sensitivity of demand, $M^{\prime}(p)$, is a sufficient statistic for measuring this gain, since the marginal social value is a known function of the co-pay when people are rational. The second term represents the reduction in insurance value for all treated individuals.

The optimal co-pay balances this trade-off, as illustrated in the following Proposition.

Proposition 1. An optimal co-pay, $p^{S}$, under the standard model exists. Supposing $p^{S} \neq 0$ and demand is positive and price-sensitive at this co-pay, i.e., $M\left(p^{S}\right)>0$ and $M^{\prime}\left(p^{S}\right)<0$, the co-pay also satisfies

$$
\frac{c-p^{S}}{p^{S}}=\frac{I}{\eta}
$$

where

$$
\eta=-\frac{M^{\prime}(p) p}{M(p)}
$$

equals the elasticity of demand for treatment.

According to Equation (8), fixing insurance value, co-pays should be higher the larger the cost

${ }^{23}$ To better interpret this expression, note that when $U$ is quadratic, $I$ can be simply expressed as $\frac{\gamma L}{E[C]}$, where $\gamma=-\frac{U^{\prime \prime}(E[C])}{U^{\prime}(E[C])} E[C]$ is the coefficient of relative risk aversion evaluated at $E[C]$ and $L=E[C]-E[C \mid m=1]$ is the magnitude of the loss. 
and elasticity of demand. ${ }^{24}$ This intuition is at the heart of rational models of copays. It suggests, for example, that copays should be lower for emergency care (where demand is less elastic) than for regular doctor's office visits (where it is presumably more sensitive). The elasticity of demand at a co-pay below cost provides a signal of the degree to which demand at that co-pay is driven by the utilization of those with a correspondingly low valuation; i.e., the extent of the moral hazard problem. The value of treatment does not enter into the standard formula: the social planner need not make this determination when patients optimally choose whether to receive care.

Proposition 1 additionally implies bounds on the optimal co-pay under the standard model. Let $p_{F I}$ denote the co-pay that would make the individual fully insured under the standard model. In other words, $p_{F I}$ is implicitly defined as the co-pay under which $I=0$, given that $m=1$ if and only if $b>p$. We will assume parameter values are such that $p_{F I}$ is unique so long as $U^{\prime \prime}<0$. For the case where $U^{\prime \prime}=0$, just pick an arbitrary value of $p$ below $c$. Note that in the special case where $b(s)=s$ for all $s$ (getting treated always returns the individual to full health), $p_{F I}=0$ whenever the individual is risk averse.

Corollary 1. Under the standard model:

1. The optimal co-pay is below cost so long as the individual is risk averse: $p^{S} \leq c$ with strict inequality if and only if $U^{\prime \prime}<0$.

2. The optimal co-pay is above the level that provides full insurance so long as demand is price-sensitive at this co-pay: $p^{S} \geq p_{F I}$ with strict inequality so long as $M^{\prime}\left(p_{F I}\right)<0$.

Corollary 1 implies that, under the standard model, optimal insurance is almost always partial. So long as the individual is risk-averse, all treatments should to some extent be covered. So long as there is moral hazard and demand is price-sensitive at low co-pays, people should bear some of the risk of needing treatment. As we will see, these qualitative features of the optimal insurance contract no longer hold in the presence of behavioral hazard.

\subsection{Behavioral Actors}

How should the social planner design insurance for behavioral actors? Consider in Figure 4 the effect of raising the price from $p<c$ to $p^{\prime}$. While in the standard model, all those whose true benefit of treatment falls between $p$ and $p^{\prime}$ no longer consume care, now those whose perceived or decision benefit of treatment falls in this range are marginal. Since decision benefit may lie below true benefit, for example when symptoms are not salient or following through is difficult, raising

\footnotetext{
${ }^{24}$ Proposition 1 does not guarantee uniqueness of the optimal co-pay. Statements regarding "the" optimal co-pay (e.g., the optimal co-pay is above $X$ ) should be read as holding for "any" optimal co-pay (e.g., any optimal co-pay is above $X$ ).
} 
the co-pay may discourage high value care. The behavioral model does not produce as simple a tradeoff between moral hazard and insurance value.

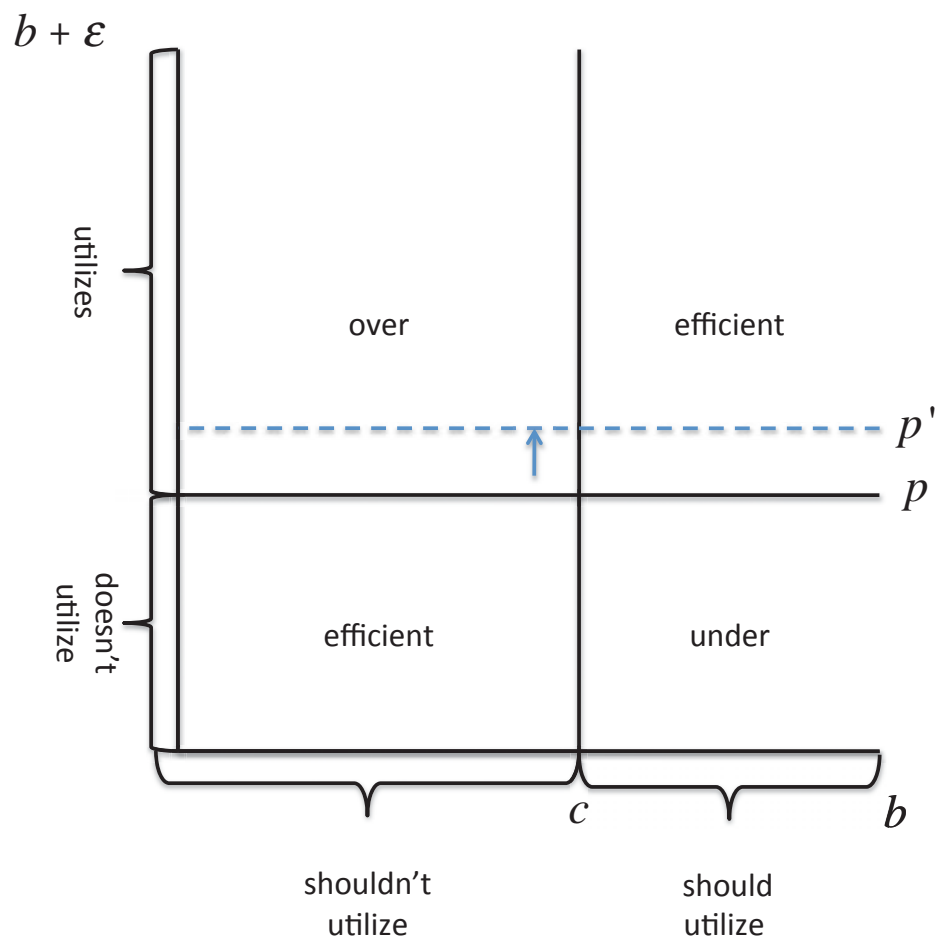

Figure 4: Effect of Changing the Co-pay on Utilization in the Behavioral Model

Differentiating $W$ with respect to $p$ subject to the break-even constraint, and normalizing yields:

$$
\frac{d W}{d p} / \frac{d W}{d y}=-M^{\prime}(p)\left(c-p+\varepsilon^{\prime}\right)-I \cdot M(p),
$$

where $\varepsilon^{\prime}=\frac{U(y-P-s)-U(y-P-s-\varepsilon)}{E\left[U^{\prime}(C)\right]}$ is an increasing function of $\varepsilon$ and should be evaluated at the disease severity of the marginal agent. ${ }^{25}$ (Note that $\varepsilon=\varepsilon^{\prime}$ for linear $U$, so we have the approximation $\varepsilon \approx \varepsilon^{\prime}$ if we take $U$ to be approximately linear). Equation (10) illustrates the discussion above. The second term again represents the reduction in insurance value for all treated individuals. The first term, $-M^{\prime}(p)\left(c-p+\varepsilon^{\prime}\right)$, captures the welfare-effect of lower utilization: it can be thought of as the number of people who are at the margin multiplied by the difference between the social cost and social value of their treatment, $\left(c-\left(p-\varepsilon^{\prime}\right)\right)$. Unlike in the standard model, the sign of this term is ambiguous. When behavioral hazard is positive, $\varepsilon^{\prime}>0$, and this term is positive: increasing the

\footnotetext{
${ }^{25}$ In other words, let $\tilde{s}$ denote the disease severity of the marginal agent: $b(\tilde{s})+\varepsilon(\tilde{s})=p$. Then $\varepsilon^{\prime}=$ $\frac{U(y-P-\tilde{s})-U(y-P-\tilde{s}-\varepsilon(\tilde{s}))}{E\left[U^{\prime}(C)\right]}$.
} 
co-pay from an amount less than cost has the benefit of decreasing overutilization. On the other hand, when behavioral hazard is negative, $\varepsilon^{\prime}<0$, and this term may be negative: increasing the co-pay can have the cost of increasing underutilization. In this case, a greater elasticity of demand may signal a greater marginal cost of raising the co-pay, rather than a greater marginal benefit as the standard model suggests.

With certain diseases, we might expect that when the co-pay is set sufficiently below cost some individuals will inefficiently receive care even with negative behavioral hazard. In this case (or with positive behavioral hazard), the optimal co-pay again trades-off the benefit of reducing demand against the cost of diminishing insurance value.

Proposition 2. An optimal co-pay, $p^{B}$, under the behavioral model exists. Supposing $p^{B} \neq 0$ and demand is positive and price-sensitive at this co-pay, i.e., $M\left(p^{B}\right)>0$ and $M^{\prime}\left(p^{B}\right)<0$, the co-pay also satisfies

$$
\frac{c-p^{B}}{p^{B}}=\frac{I}{\eta}-\frac{\varepsilon^{\prime}}{p^{B}} .
$$

There are two key implications of Equation (11). First, co-pays should vary across treatments with similar demand elasticities, unlike in the standard model where the elasticity of demand is a sufficient statistic for the optimal co-pay (fixing cost $c$ and insurance value $I$ ). To illustrate, it may be optimal to vary co-pays across classes of drugs with similar costs and demand elasticities if we expect drugs across these classes to be differentially misvalued. Despite having similar demand elasticities, it is possible that co-pays for drugs treating chronic conditions (e.g., antidiabetics) should be lower than co-pays for symptom-alleviating drugs (e.g., pain medications), for example. While advocated by some health researchers (for example, Chernew et al. 2007), such differential cost-sharing is a rarity in practice, as illustrated in Table 2. We will return to the question of why competitive markets may fail to result in efficient outcomes following the positive analysis of Section 6.

A second implication of Equation (11) is that health "insurance" is no longer just about financial protection: it can also result in more efficient health care delivery. As noted above, newly insured patients consume much more high-value, low-cost care - a jump unlikely to be solely driven by the change in prices and relaxation of credit constraints that come with insurance. Even when individuals are risk-neutral and there is no value to financial insurance $(I=0)$, Equation (11) indicates that the optimal co-pay can differ from cost to provide behavioral consumers with incentives for more efficient utilization decisions. ${ }^{26}$ In fact, when consumers are risk-neutral and

\footnotetext{
${ }^{26}$ In this spirit, some insurers have begun to experiment with incentivizing patients to take their medications, as reported in a recent New York Times article (http://www.nytimes.com/2010/06/14/health/14meds.html). See also Volpp et al. (2009).
} 
biased $(\varepsilon(s) \neq 0$ for all $s$ ), the extent of behavioral hazard (at the margin) fully determines the optimal co-pay, which differs from cost and maximizes health net of costs, or $\mathcal{E}(p)$.

Proposition 2 implies that optimal co-pays may be outside the bounds implied by the standard model.

\section{Corollary 2. Under the behavioral model:}

1. The optimal co-pay may be above cost even when the individual is risk averse: can have $p^{B} \geq$ ceven when $U^{\prime \prime}<0$.

2. The optimal co-pay may be below the level that provides full insurance even if demand is price-sensitive at this co-pay: can have $p^{B} \leq p_{F I}$ even when $M^{\prime}\left(p_{F I}\right)<0$.

Corollary 2 implies that, under the behavioral model, optimal insurance need not be partial. ${ }^{27}$ If positive behavioral hazard is sufficiently large, it can be optimal for the insurance company to deny coverage of certain treatments, even if they benefit some risk-averse individuals. If negative behavioral hazard is sufficiently large, it can be optimal for the insurance company to fully cover (or even subsidize) treatments, even when demand is price sensitive.

It is important to emphasize that both behavioral and moral hazard vary across conditions and can be forces that work in the same direction or opposite directions in jointly determining the optimal co-pay. In the case of blood pressure medication, for example, moral hazard may drive use up (increasing the optimal copay) while negative behavioral hazard drives use down (reducing the optimal copay). On the other hand, in the case of MRIs for back pain, both moral hazard and positive behavioral hazard drive use up (increasing the optimal copay). Finally, take the case of setting a broken bone (a high value, hard-to-overdo procedure). In this situation, there may be little scope for moral hazard $(b(\underline{s})>c)$ and thus no case for positive copays, even absent behavioral hazard.

\section{Nudges}

In Section 6 we consider some properties of the optimal plan when nudges are available, and compare it to the plan provided by the market. ${ }^{28}$ For now, we merely point out that the optimum may involve nudging that encourages care. For example, fix the co-pay at the optimal level under

\footnotetext{
${ }^{27}$ To be precise, the amount of insurance coverage is measured with respect to the degree to which people would be insured if they behaved according to the standard model. For example, if $b(s)=s$ for all $s$, then we say the agent is fully covered when $p=0$ because in this case his marginal utility would be independent of disease severity if he acted in accordance with the $b>p$ choice rule. Note that in this example the agent's marginal utility may be still be stochastic if he sometimes fails to get treated at zero co-pays due to negative behavioral hazard.

${ }^{28} \mathrm{~A}$ detailed analysis of optimal nudging in more general policy problems can be found in Mullainathan, Schwartzstein and Congdon (2012).
} 
the default nudge. A sufficient condition for it to be better to nudge in a way that encourages care is roughly that such nudging results in health benefits that outweigh direct costs of greater demand plus any costs of implementing the nudge. ${ }^{29}$

\subsection{Interpreting the Evidence}

These results (particularly Equation (10)) highlight an important point for empirical work. They suggest that simply looking at demand responses alone can provide a misleading impression. When there is merely moral hazard, the overall demand response is a powerful tool for measuring the welfare impact of the changes in utilization driven by co-pay changes. When there is behavioral hazard, this composite response is harder to interpret. For example, a high response might indicate a great deal of moral hazard (and hence a cost of providing insurance) or could indicate a great deal of negative behavioral hazard or price-responsive underutilization (and hence an additional benefit to insurance).

There are many empirical estimates of these aggregate demand responses. When one looks closer, these aggregate responses hide a great deal of diversity, some of which is consistent with behavioral hazard. In fact, there is evidence that demand for "effective care" is often as elastic as demand for "ineffective care" - as summarized in Table 3. A quasi-experimental study of the effects of small increases in copayments (rising from around \$1 to around \$8) among retirees in California by Chandra, Gruber, and McKnight (2010) suggests that HMO enrollees' elasticity for "lifestyle drugs" such as cold remedies and acne medication is virtually the same as for acute care drugs such as anti-convulsants and critical disease management drugs such as beta-blockers and statins - all clustered around -0.15 (2007; unpublished details provided by authors, summarized in Table 4). Johnson et al. (1997) find that small drug copay increases (rising from 50\% coinsurance up to a max of $\$ 25$ to a $70 \%$ coinsurance up to a max of $\$ 30$ ) drove reductions of $40 \%$ in both anti-asthma medications and in mild analgesics. Adherence to antidiabetic medications is also quite price sensitive and reductions in adherence are associated with greater rates of amputation, ulcers, and short-term disability days (Gibson et al. 2010; Goldman et al. 2004). Analysis of the RAND health insurance experiment found that cost-sharing induced the same $40 \%$ reduction in beta blockers as it did in cold remedies - with reductions in drugs deemed "essential" on average the same as those deemed "less essential" (Lohr et al., 1986).

Much of the "effective care" that is reduced with the introduction of small increases in low copays seems likely to be indicative of behavioral hazard rather than moral hazard - and can lead to

\footnotetext{
${ }^{29}$ Nudges that reduce demand can also be useful when there is positive behavioral hazard, since such nudges can curb overuse without undermining insurance value. It is tempting to suppose that nudging in a way that eliminates errors is always optimal when such nudging is possible and does not have direct costs. However, such debiasing can undermine broader social welfare in cases when it increases demand and, with it, the moral hazard cost of insurance (Mullainathan et al. 2012, Pauly and Blavin 2008).
} 
serious health consequences (as summarized in Table 5). The reduction in high-value care shown in Chandra et al. (2010) is associated with increases in subsequent hospitalizations. Similarly, Hsu et al. (2006) found that the imposition of a cap on Medicare drug benefits reduced adherence to chronic disease medications and was associated with a subsequent increase in hospitalizations. Selby et al. (1996) find that the imposition of a \$25-\$35 emergency room copay resulted in a decrease of emergency room utilization in truly emergency situations (such as cardiac arrest, stroke, heart attack, appendicitis, or respiratory failure) of almost 10\%. A number of studies (Chernew, 2008; Goldman, 2006; Landsman, 2005; Johnson, 1997; Lohr, 1986) show reductions in use of statins, anti-depressants, beta blockers, or asthma medications of known efficacy associated with even small increases in low copayments. Beta blockers, for example, can reduce post-heart attack mortality by 20-30\% (Yusuf et al., 1985), and statins can reduce the risk of events like heart attacks by $25 \%$ (Zhou et al., 2006).

In light of this empirical evidence, optimal copays cannot be determined solely using demand responses to co-pay changes. Rather, Proposition 2 highlights the need to understand the degree to which people make mistakes on the margin - the marginal behavioral error (or internality), $\varepsilon$.

\section{Empirical Implementation}

\subsection{Using Estimates of the (Marginal) Health Value of Care: A Foundation for Value-Based Insurance Design}

Several methods for estimating the marginal behavioral error (or internality), $\varepsilon$, are available. First, we consider how this error can be estimated by comparing health and demand responses to higher co-pays.

Proposition 3. Consider a co-pay, $p$, at which demand is price-sensitive, so $M^{\prime}(p)<0$. Let $s(p)$ denote the disease severity of the marginal agent given the co-pay and let $\varepsilon(p) \equiv \varepsilon(s(p))$ denote the behavioral error of the marginal agent. The following relationship holds between the marginal behavioral error, demand responses, and health responses:

$$
\varepsilon(p)=p-\frac{H^{\prime}(p)}{M^{\prime}(p)} .
$$

From Equation (12), we see that behavioral hazard is negative at the margin whenever there is a positive net return to the last private dollar spent on treatment, and is positive otherwise. ${ }^{30} \mathrm{~A}$ slightly more complicated formula holds in more general models allowing for heterogeneity (see

\footnotetext{
${ }^{30}$ To clarify, for $p>0$, Equation (12) is negative whenever $\frac{\varepsilon(p)}{p}<0$ which is true if and only if $\frac{1}{p} \cdot \frac{H^{\prime}(p)}{M^{\prime}(p)}-1>0$, i.e., if and only if there is a positive net return to the last private dollar spent on treatment.
} 
Proposition 8 in Appendix B), but that formula has identical implications for understanding the welfare impact of raising the co-pay and for setting optimal co-pays.

Proposition 3 can be used to estimate the welfare impact of raising the co-pay and to calibrate the optimal co-pay formula. Plugging (12) into (10) and making the approximation $\varepsilon \approx \varepsilon^{\prime}$ yields

$$
\frac{d W}{d p} / \frac{d W}{d y} \approx-M^{\prime}(p)\left[c-\frac{H^{\prime}(p)}{M^{\prime}(p)}\right]-I \cdot M(p) .
$$

In the standard model, Equation (13) reduces to (7) (because, in the standard model, $\left|H^{\prime}(p)\right| /\left|M^{\prime}(p)\right|=$ $p)$, and the first term of (13) is positive because there is a negative net return to the last social dollar spent on treatment (assuming $p<c$ ). ${ }^{31}$ More generally, the first term of (13) is positive if and only if there is in fact a negative net return to the last social dollar spent on treatment; i.e., increasing the co-pay results in social cost savings that exceed the monetary value of health losses. Otherwise, raising the co-pay is costly both because it reduces insurance value and utilization. ${ }^{32}$

Turning to the optimal co-pay, we make the approximation $\varepsilon \approx \varepsilon^{\prime}$ and plug (13) into (11), yielding

$$
\frac{c-p}{p} \approx \frac{I}{\eta}+\left(\frac{H^{\prime}(p)}{p M^{\prime}(p)}-1\right) .
$$

From Equation (14) we roughly have that co-pays should be lower (higher) than traditionally argued if increasing the co-pay tends to result in a reduction of consumer spending on health that is lower (greater) than the associated dollar value of health losses. Put another way, co-pays should be lower than traditionally argued when there is a positive net return to the last private dollar spent on treatment and should be adjusted according to the magnitude of this net return, $\left|H^{\prime}(p)\right| /\left(p\left|M^{\prime}(p)\right|\right)-1$.

Note that, unlike in the standard model, the value of treatment now enters into the determination of the optimal co-pay insofar as it influences $H^{\prime}(p)$. For a given demand response to co-pays, co-pays should be lower when this demand response has greater adverse effects on health. This response should be estimated treatment by treatment. Thus, it is possible that copays should be lower for routine maintenance visits for people with chronic diseases than for general doctor's

\footnotetext{
${ }^{31}$ In the standard model, the net return on the last social dollar spent on treatment equals $\frac{1}{c} \cdot \frac{H^{\prime}(p)}{M^{\prime}(p)}-1=\frac{p-c}{c}$.

${ }^{32}$ Standard estimates of the welfare loss of "excess health insurance" fail to take into account the deviation between $\left|H^{\prime}(p)\right| /\left|M^{\prime}(p)\right|$ and $p$ (e.g., Feldstein 1973, Feldman and Dowd 1991). When, over the relevant range, the first term exceeds the second (health responses are quite big), standard estimates of the welfare benefit of setting a higher co-pay will be too large. In particular, if insurance value is small (e.g., because of low levels of risk aversion), then standard estimates tend to suggest a welfare gain to reducing insurance coverage from existing levels whenever demand is sufficiently elastic. In a more flexible model, this finding would be reversed for treatments where the net return to the last social dollar spent on treatment is positive over the relevant range.
} 
office visits (Newhouse 2006). More broadly, behavioral hazard provides a foundation for valuebased insurance design (Chernew et al. 2007, Pauly and Blavin 2008), where, all else equal, cost sharing is lower for higher value care.

Note also that equations (13) and (14) are robust to more general assumptions about the nature of insurance contracts and biases. As demonstrated in Appendix B, these equations continue to hold even when (i) a co-pay change affects demand for a broad category of treatments (e.g., the insurer faces a prohibitively high administrative cost to differentiating between many treatments), (ii) biases are heterogeneous across the population (e.g., some people are more present-biased than others), and (iii) people may simply be forgetful in deciding whether to get treated.

\section{An Empirical Example}

We draw on Choudhry et al.'s (2011) work on the effects of eliminating copays for recent heart attack victims to illustrate the potential importance of taking behavioral hazard into account. ${ }^{33}$ They randomly assigned patients discharged after heart attacks to a control group with usual coverage (with copayments in the \$12-\$20 range) or a treatment group with no co-payments for statins, beta blockers and ACE inhibitors (drugs of known efficacy), and tracked adherence rates and clinical outcomes over the next year. Faced with lower prices, consumers used more drugs: the full coverage group was significantly more adherent to their medications, using on average \$106 more worth of cardiovascular-specific prescription drugs.

Under the moral hazard model, this fact alone tells us the health consequences of eliminating copays. Rational patients forgo only care with marginal value less than their out-of-pocket price. The average patient share under usual coverage in the Choudry data is about $25 \%$, implying that the extra care consumed when copays are eliminated has a monetized health value of at most $\$ .25$ on the dollar. Given the $\$ 106$ increase in spending, the moral hazard model then predicts a health impact of at most $\$ 106 \cdot .25=\$ 26.50$ per patient. This in turn implies a moral hazard welfare loss from eliminating copayments of at least $\$ 106(1-.25)=\$ 79.50$ per person. In other words, the $\$ 106$ increase in spending is comprised of $\$ 26.50$ of health value plus $\$ 79.50$ of excess utilization. This is the kind of exercise routinely performed with demand data. ${ }^{34}$

But Choudhry et al. collected data on health impacts, which we can use to gauge the performance of the moral hazard model by comparing the implied health benefits with the observed ones.

\footnotetext{
${ }^{33}$ We use this particular study because it measures not only demand responses, but also a rich variety of health responses. While the setting is admittedly quite specific, we believe the qualitative conclusions are illustrative for broader populations and treatments. Appendix $\mathrm{C}$ provides a stylized example using the case study of treatment for high blood pressure, though it is difficult to perform a rigorous analysis given data limitations.

${ }^{34}$ Given the standard assumption that people have linear demand curves, we can derive a tighter lower bound on the welfare loss. In this case, the moral hazard model implies a welfare loss of at least $\$ 106(1-.25 / 2)=\$ 92.75$ (see, e.g., Feldman and Dowd 1991).
} 
The increase in prescription drug use was associated with significantly improved clinical outcomes: patients in the full coverage group exhibited lower rates of vascular events (1.8 percentage points), myocardial infarction (1.1 percentage points), and death from cardiovascular causes (.3 percentage points). We apply the commonly used estimate of a $\$ 1$ million value of a statistical life to the reduction in the mortality to get a crude measure of the dollar value of health improvements. ${ }^{35}$ This implies that the elimination of copays leading to a 3 percentage point reduction in mortality generates a value of $\$ 3,000$. This $\$ 3,000$ improvement substantially exceeds the standard model's prediction of $\$ 26.50$, suggesting large negative behavioral hazard. ${ }^{36}$

This also suggests that we need a very different welfare calculation. In the presence of behavioral hazard, we need to use an estimate of the marginal private health benefit to calculate welfare. To perform a rough back-of-the-envelope calculation, take the $\$ 3,000$ figure from above. The $\$ 106$ increase in spending then generates a surplus of \$2,894 per person (a gross return of \$28 per dollar spent).The presence of behavioral hazard thus reverses how we interpret the demand response to eliminating copayments in this example: Moral hazard implies a welfare loss, while behavioral hazard implies a gain that is over 30 times larger in magnitude. ${ }^{37}$ In both models, of course, lowering copays also increases the insurance value of the plan.

This example illustrates the importance of having estimates of both demand responses and health responses to changes in prices. This information is not available for all services, in part because of the difficulty in linking clinical and administrative data (Goldman and Joyce 2012). It would certainly be daunting to perform an exhaustive list of experiments and calculations, but it is worth noting that a small number of conditions account for a large share of health spending. In particular, the areas in which we observe substantial underutilization - such as management of chronic diseases like diabetes, high blood pressure, asthma, and high cholesterol - are responsible for a substantial share of total health care utilization. Patients with circulatory system conditions like high blood pressure and high cholesterol are responsible for $17 \%$ of total health care spending; mental conditions like depression for another $9 \%$; respiratory conditions like asthma another $6 \%$;

\footnotetext{
${ }^{35}$ This calculation is admittedly crude, but provides an illustrative example. Estimates of the value of a statistical life clearly vary based on the age at which death is averted and the life expectancy gained - averting the death of a young healthy worker might be valued at $\$ 5$ million - and mortality is only one aspect of the potential changes in health. While the estimated reduction in mortality is not statistically significant at conventional levels, the other health impacts are. We focus on the mortality reduction because it is easiest to monetize.

${ }^{36}$ Making this point in a slightly different way, applying the traditional moral hazard calculus in this situation implies that people place an unrealistically low valuation on their life and health.

${ }^{37}$ As in basic moral hazard calculations, this analysis ignores substitution between treatments. In this example, total spending (prescription drug plus nondrug) went down by a small, non-statistically significant amount when co-payments were eliminated on preventive medications after heart attack, as did insurer costs. Taking these nonsignificant offset effects at face value would imply that welfare goes up even before taking behavioral hazard into account (Glazer and McGuire 2012), though it raises a puzzle as to why private insurers did not reduce co-pays on their own. However, even in this case, incorporating behavioral hazard substantially changes the analysis by providing a strong rationale for reducing the co-pay that does not rely on offsetting cost savings.
} 
and endocrine conditions like diabetes another 4\% (Roehrig et al., 2009). This means that a limited number of studies linking price changes, demand changes, and health changes could go a long way.

Even absent such multi-step evidence, however, we can glean some benchmarks about the potential benefits of optimal copayment design in a model with both moral hazard and behavioral hazard from the literature that looks at particular steps in this chain. This requires applying results from one particular step (e.g. the effect of a co-pay change on one measure of adherence, such as refilling prescriptions) generated from a particular marginal population to the next step (e.g. effect of a different measure of adherence, such as missed pills, on heart attacks) generated in a different setting with a different population, over a different time frame, etc. Appendix C provides a very stylized example using the case study of treatment for high blood pressure. High blood pressure afflicts 68 million adults in the U.S. (CDC Vital Signs 2011) and is an important driver of overall health care costs. Anti-hypertensive drugs are effective, but adherence is only between 50 and 70 percent, suggesting negative behavioral hazard. Using existing estimates, we show that small reductions in copays increase compliance with anti-hypertensive therapy and that better compliance generates substantial health gains. This implies a large net return on the marginal social dollar spent on improving blood-pressure medication adherence - and that the failure of existing plans to address behavioral hazard could be generating large costs.

\section{Potential Challenges}

Of course, a challenge to empirically implementing Equation (14) is that portions of the health response may be unobservable, or difficult to map to hedonic benefits. It may be possible to estimate the degree to which taking a pill reduces the risk of mortality and translate this into (money-metric) utility; it may be more difficult to estimate certain side-effects, or the inconvenience of treatment. In some instances, however, we may have enough information to confidently bound the unobservable component in which case we can still say something about the sign and possibly the magnitude of the behavioral error.

To illustrate, decompose the change in health per marginal change in demand into observable and unobservable components:

$$
\frac{H^{\prime}(p)}{M^{\prime}(p)}=h_{O}(p)+h_{U}(p),
$$

where $h_{O}(p)$ represents the observable component, and $h_{U}(p)$ the unobservable component. For example, the observable component could include a proxy for quality-adjusted life years gained per marginal filled prescription and the unobservable component could include non-pecuniary costs (e.g., side-effects) associated with filling the prescription (all in dollars). If we can bound the unobservable component as belonging to $\left[\underline{\left.h_{U}(p), \overline{h_{U}(p)}\right]}\right.$, then we can also bound the behavioral 
error:

$$
\varepsilon(p) \in\left[p-\left(h_{O}(p)+\overline{h_{U}(p)}\right), p-\left(h_{O}(p)+\underline{h_{U}(p)}\right)\right] .
$$

Bound (16) can be useful in giving a sense of the welfare impact of changing the co-pay or even in pinning down the optimal co-pay if it is constrained (e.g., to lie in $[0, c]$ ) when the magnitude of the observable health change is large relative to the uncertainty about the unobservable component. This is more likely to be the case when considering certain highly efficacious treatment regimins with few suspected painful side effects (e.g., treatments listed in Table 1) than in considering treatments with direct non-pecuniary costs that may be experienced quite differently across people (e.g., colonoscopies). Returning to the Choudhry et al. (2011) example, it seems unlikely that the cost of unobserved side-effects of statins, beta blockers, and ACE-inhibitors is anywhere near $\$ 2894$ for a given patient in a year, so taking these effects into account should not reverse the conclusion that eliminating co-payments leads to a welfare gain.

This bound is also helpful in illustrating where the logic of this framework is on slippery ground. Take the case of caloric and unhealthy ("junk") foods. The net benefits of these foods consists of both the long term health costs of eating these foods and the short term benefits they provide. While the health costs of the marginal agent are plausibly estimable, the short term benefits are not (absent a structural model). Trying to apply the bounding exercise above to junk foods would only be plausible to the extent that one can rule out that individuals do not receive as much idiosyncratic pleasure as the measured portion of the internality. This is not to say that there are not internalities, merely that in cases such as these (and others) we may not be able to effectively use the bounds to build a reasonable case.

In such instances, other data or prior information may be useful. One approach is to examine demand responses to nudges.

\subsection{Other Approaches}

\section{Using Nudge Responses}

Nudges (e.g., text message reminders) can also be used to help estimate the marginal behavioral error, so long as there is some belief that a nudge influences demand through affecting the agent's marginal valuation, as illustrated in the following proposition.

\section{Proposition 4.}

1. (Negative behavioral hazard). Suppose we know that $\varepsilon_{0} \leq 0$ and $\varepsilon_{n} \leq 0$ for all s. Further, let $p_{0}, p_{n}$ be such that $p_{0}<p_{n}$, but $0<M_{0}\left(p_{0}\right) \leq M_{n}\left(p_{n}\right)<q$. Then,

$$
-\varepsilon_{0}\left(p_{0}\right) \geq p_{n}-p_{0}
$$


where $\varepsilon_{0}\left(p_{0}\right) \equiv \varepsilon_{0}\left(s\left(p_{0}\right)\right)$ is the behavioral error evaluated at the disease severity of the marginal agent given co-pay $p_{0}$ and nudge $n=0$.

2. (Positive behavioral hazard). Alternatively, suppose we know that $\varepsilon_{0} \geq 0$ and $\varepsilon_{n} \geq 0$ for all s. Further, let $p_{0}, p_{n}$ be such that $p_{n}<p_{0}$, but $0<M_{n}\left(p_{n}\right) \leq M_{0}\left(p_{0}\right)<q$. Then,

$$
\varepsilon_{0}\left(p_{0}\right) \geq p_{0}-p_{n}
$$

To illustrate how Proposition 4 can be used to estimate $\varepsilon_{0}$, suppose a researcher is convinced that a treatment is undervalued, and he has access to nudge $n$ which he believes brings decision utility more in line with hedonic utility. Bound (17) can be applied whenever demand is interior and $M_{0}\left(p_{0}\right) \leq M_{n}\left(p_{n}\right)$. The latter condition can be re-written as

$$
\underbrace{M_{n}\left(p_{0}\right)-M_{0}\left(p_{0}\right)}_{\text {mand response to nudge }}-\underbrace{\left[M_{n}\left(p_{0}\right)-M_{n}\left(p_{n}\right)\right]}_{\text {demand response to co-pay change }} \geq 0,
$$

so bound (17) can be applied whenever the demand response to an increase in co-pay from $p_{0}$ to $p_{n}$, fixing the nudge at $n$, is lower in magnitude than the demand response to the nudge, fixing the co-pay at $p_{0}$. Thus, the tightest lower bound a researcher can estimate for $-\varepsilon_{0}\left(p_{0}\right)$ is $\tilde{p}_{n}-p_{0}$, where $\tilde{p}_{n}$ is the value of $p_{n}$ that equates the demand response to an increase in co-pay to the demand response to the nudge; i.e., it is the value that makes (19) hold with equality.

This discussion points to an empirical strategy of how to estimate the degree to which treatments are misutilized and therefore how insurance should be designed. Even if some nudge is not a cost-effective means of promoting better health choices when scaled up, it may nonetheless be useful to implement the nudge on a small scale to estimate structural parameters of the behavioral model through comparing demand sensitivity to the nudge with sensitivity to co-pay changes.

\section{Using Positive Models of Behavior}

Confidence in specific, positive, assumptions about utility and behavior can also be useful (Beshears et al. 2008, Mullainathan et al. 2012). For example, if the marginal internality is thought to arise from present-bias, then knowledge of the degree to which benefits or costs of treatment are immediate or delayed can give a sense of which treatments should have higher or lower co-pays.

These models can also be useful in quantitatively estimating $\varepsilon$ and, in turn, the optimal co-pay. For example, in the case of present-bias, we showed before that $\varepsilon(s)=-(1-\beta) v(s)$, where $\beta \leq 1$ captures the degree of present-bias and $v(s)$ represents delayed benefits of treatment. This reduces the problem of estimating the marginal internality to estimating the degree of present-bias, $\beta$, and the delayed benefits (or costs) of treatment. If we are confident that estimates of present- 
bias transfer well from one context to another, then this implies that we can estimate the marginal internality without directly estimating current benefits or costs to treatment, an approach that is particularly helpful when these terms are the hardest to pin down (e.g., the disutility from getting a colonoscopy) and we can use previous estimates of $\beta$ (for example, taken from Laibson et al. 2006). ${ }^{38}$

\section{What Will the Market Provide?}

The analysis in Section 4 addressed the normative question of how we should design health insurance. We now address the companion positive question of what the market will actually provide. Doing so will allow us to analyze whether the equilibrium insurance contract in fact results in more efficient health delivery through counteracting biases (as compared to a world without health insurance), and whether there is room for welfare-improving government intervention.

\subsection{Basic Model}

We derive the equilibrium insurance contract in a competitive market. This contract may differ from the optimal contract because people may not fully understand how they are biased and misforecast their demand for treatment. Formally, define $\hat{m}(p)$ to be an individual's forecasted demand for care at a given price: it equals 1 if and only if $b(s)+\hat{\varepsilon}(s)>p$, where $\hat{\varepsilon}(s)$ represents the forecasted wedge between decision and hedonic valuations. At the time of contracting, the agent may not appreciate that she will undervalue the need to take a chronic disease medication or that she will seek out any treatment for back pain. Define forecasted aggregate demand to equal $\hat{M}(p)=E[\hat{m}(p)]$. In common with much of the behavioral literature, we will highlight two extreme cases: the case where the agent perfectly understands her biases and the case where she thinks she is unbiased at the time of contracting. Formally, the agent is said to be sophisticated when $\hat{\varepsilon}(s)=\varepsilon(s)$ for all $s$ and to be naive when $\hat{\varepsilon}(s)=0$ for all $s{ }^{39}$

In equilibrium, the market will supply insurance plan $(P, p)$ to maximize the agent's perceived

\footnotetext{
${ }^{38}$ To take a related example, Gruber and Koszegi (2001) calibrate the optimal tax per pack of cigarettes through estimating the health consequences of smoking, together with making assumptions about the degree of present bias.

${ }^{39}$ There is another potential form of naivete, namely that behavioral agents misperceive treatment benefits at the time of contracting, not just at the time of making a treatment decision (i.e., at the time of contracting, they misforecast the benefit of getting treated as $b(s)+\varepsilon(s)$ given severity $s$ ). It would be natural to assume this form of naivete if decision errors are the result of incorrect beliefs about treatment benefits, for example, but not if these errors are the result of biases such as procrastination. We abstract from this form of naivete to limit the number of cases considered. However, we note that it is particularly easy to characterize the equilibrium outcome given this form of naivete: it is just the solution to the planner's problem if the planner mistakenly perceives treatment benefits as equaling $\hat{b}(s)=b(s)+\varepsilon(s)$ for all $s$. That is, the equilibrium co-pay would satisfy (8).
} 
expected utility

$$
\hat{W}=(1-q) U(y-P)+q E[U(y-P-s+\hat{m}(b-p)) \mid \text { sick }]
$$

subject to zero profit constraint ${ }^{40}$

$$
P=M(p)(c-p)
$$

The agent's perceived expected utility $\hat{W}$ can differ from her actual utility $W$ because she may misforecast her demand for treatment.

Since the optimal contract maximizes $W$ subject to (21), it is immediate that the optimal and equilibrium contract coincide when the agent is sophisticated. To derive the terms of the contract for the more general case, totally differentiate $\hat{W}$ subject to (21) with respect to $p$ and normalize:

$$
\begin{aligned}
\frac{d \hat{W}}{d p} / \frac{d \hat{W}}{d y} & =-\hat{M}^{\prime}(p)\left(c-p+\hat{\varepsilon}^{\prime}\right)-\hat{I} \hat{M}(p) \\
& +\left(\left|M^{\prime}(p)\right|-\left|\hat{M}^{\prime}(p)\right|\right)(c-p)+(M(p)-\hat{M}(p)),
\end{aligned}
$$

where $\hat{I}=\left(\frac{E\left[U^{\prime}(C) \mid \hat{m}(p)=1\right]-E\left[U^{\prime}(\hat{C})\right]}{E\left[U^{\prime}(\hat{C})\right]}\right)$ equals the forecasted insurance value to consumers $(\hat{C}=$ $y-P-s+\hat{m}(b-p))$ and $\hat{\varepsilon}^{\prime}=\frac{U(y-P-s)-U(y-P-s-\hat{\varepsilon})}{E\left[U^{\prime}(\hat{C})\right]}$ is an increasing function of $\hat{\varepsilon}$ and should be evaluated at the forecasted marginal disease severity. ${ }^{41}$

The terms on the first line of (22) just equal the perceived counterparts of those that make up the true welfare effect of a higher co-pay: the first is the perceived welfare effect of lower utilization and the second is the perceived reduction in insurance value. The terms on the second line are more interesting. These arise because the agent may misforecast demand and consequently view the tradeoff between a higher co-pay and a lower premium as being more or less favorable than it actually is. Formally, the zero profit condition means that the equilibrium insurance contract is actuarially fair. The premium thus goes down by $-M^{\prime}(p)(c-p)+M(p)$ for every marginal

${ }^{40}$ As is well known, the solution to (20) and (21) will coincide with the outcome when insurers maximize profits

$$
\max _{P, p} P-M(p)(c-p)
$$

subject to an "individual rationality" constraint for the representitive agent

$$
\hat{W} \geq \bar{u}
$$

where $\bar{u}$ is such that the solution to this problem yields zero profits. In fact, the formula we derive for the equilibrium co-pay holds for any $\bar{u}$, so the degree of market power does not change the logic of how co-pays will be set in equilibrium.

${ }^{41}$ In other words, let $\hat{s}$ denote the forecasted marginal disease severity: $b(\hat{s})+\hat{\varepsilon}(\hat{s})=p$. Then $\hat{\varepsilon}^{\prime}=$ $\frac{U(y-P-\hat{s})-U(y-P-\hat{s}-\hat{\varepsilon}(\hat{s}))}{E\left[U^{\prime}(\hat{C})\right]}$. 
increase in the co-pay. However, the agent believes that it should go down by $-\hat{M}^{\prime}(p)(c-p)+$ $\hat{M}(p)$. Differencing these two expressions gives the second line of (22). Overforecasting the degree to which the premium should go down reduces the apparent desirability of raising the copay; underforecasting raises the apparent desirability.

Setting (22) equal to zero produces a formula for the equilibrium co-pay.

Proposition 5. An equilibrium co-pay, $p^{E}$, under the behavioral model exists. Supposing $p^{E} \neq 0$, and demand as well as forecasted demand are positive and price-sensitive at this co-pay, the copay also satisfies

$$
\frac{c-p^{E}}{p^{E}}=\frac{\hat{M}^{\prime}\left(p^{E}\right)}{M^{\prime}\left(p^{E}\right)}\left[\frac{\hat{I}}{\hat{\eta}}-\frac{\hat{\varepsilon}^{\prime}}{p^{E}}\right]+\frac{\left(\hat{M}\left(p^{E}\right)-M\left(p^{E}\right)\right)}{p^{E}\left|M^{\prime}\left(p^{E}\right)\right|} .
$$

Welfare under $p^{E}$ is (weakly) lower than welfare under $p^{B}$.

Comparing (23) with (11) shows that the equilibrium co-pay differs from the optimal co-pay because the agent misforecasts demand: there is a market failure resulting from lack of sophistication.

To gain more intuition for how this works, consider the simple case of a risk-neutral naive agent (so $I=\hat{I}=0, \varepsilon^{\prime}=\varepsilon$, and $\hat{\varepsilon}=\hat{\varepsilon}^{\prime}=0$ ). In this case, the optimal co-pay formula reduces to

$$
p^{B}=c+\varepsilon\left(s\left(p^{B}\right)\right)
$$

where $s(p)$ denotes the disease severity of the marginal agent given co-pay $p$. Note that, at the optimal co-pay, the marginal agent fully internalizes her "internality" (the difference between $p$ and $c$ acts as a Pigouvian tax set at the level of the marginal internality $\varepsilon(s(p))) .{ }^{42}$ Additionally, this co-pay maximizes health net of costs, or $\mathcal{E}(p)$.

On the other hand, the equilibrium co-pay in this case satisfies

$$
p^{E}=c+\frac{M\left(p^{E}\right)-\hat{M}\left(p^{E}\right)}{\left|M^{\prime}\left(p^{E}\right)\right|} .
$$

Rather than being determined by the level of the marginal internality, the equilibrium co-pay is set according to the difference between actual and predicted demand: the larger this difference, the

\footnotetext{
${ }^{42}$ Since the optimal co-pay in this case acts as a Pigouvian tax to get the agent to internalize his internality, it is tempting to think that the analysis would be the same if we were to think of $\varepsilon$ as an externality rather than an internality. However, there are important differences. If $\varepsilon$ were an externality then market forces alone would not correct the problem (Coasian bargaining seems unlikely in this setting) - government intervention would be necessary. However, when people are sophisticated, market forces can correct an internality. Likewise, even when people are naive, market forces can lead to an equilibrium in which agents partially internalize their internality, as we will soon see.
} 
greater the co-pay. Since, generically, these terms do not coincide, the equilibrium co-pay tends to result in inefficient levels of health $\left(\mathcal{E}\left(p^{E}\right)<\mathcal{E}\left(p^{B}\right)\right)$ : There is a social loss from equilibrium insurance not being value-based.

However, the difference between co-pay and cost will go in the right direction. With negative behavioral hazard $(\varepsilon(s)<0$ for all $s)$, the optimal co-pay is set below the cost of care to encourage demand, $p^{B}<c$. In this case the equilibrium co-pay will also lie below the cost of care, $p^{E}<c$, but for an entirely different reason: a naif overestimates his own demand and views the tradeoff between lower co-pays and higher premiums as more favorable than it actually is. ${ }^{43}$ (By a symmetric argument, with positive behavioral hazard, $p^{B}>c$ and $p^{E}>c$.) A way to see this intuition even more sharply is when agents are inattentive and forget to get treated with some probability that is independent of decision benefits and costs, a case formally considered in Appendix B. The optimal copay does not depend on the forgetting probability because this probability does not influence decisions at the margin. Yet, at the same time, forgetfulness leads agents to overforecast their usage and, in equilibirium, profit maximizing firms will have an incentive to lower the copay in response to this bias.

\subsection{Nudges}

Suppose insurers are able to use nudges. Define $\hat{m}_{n}(p)$ as the individual's forecasted demand for care at a given price conditional on nudge $n: \hat{m}_{n}(p)$ equals 1 if and only if $b(s)+\hat{\varepsilon}_{n}(s)>p$. Let $\hat{M}_{n}(p) \equiv E\left[\hat{m}_{n}(p)\right]$ equal forecasted aggregate demand given a nudge. For a sophisticated consumer, $\hat{\varepsilon}_{n}=\varepsilon_{n}$ for all $n$ : sophisticates completely understand how nudges will affect their decisions. For a naive consumer, $\hat{\varepsilon}_{n}=0$ for all $n$ : naifs think their decisions will be independent of nudges. Naifs do not appreciate that reminders and educational programs will improve adherence, or that small hassel factors will greatly influence whether they seek treatment.

We assume that nudges can be contracted over. In equilibrium, the market will supply insurance plan $(P, p, n)$ to maximize the agent's perceived expected utility

$$
\hat{W}=(1-q) U(y-P)+q E\left[U\left(y-P-s+\hat{m}_{n}(b-p)\right) \mid \text { sick }\right]
$$

\footnotetext{
${ }^{43}$ This is analagous to why profit-maximizing firms may charge high up-front fees and below-cost usage prices for "investment goods" when consumers are partially naive about their self-control problems (DellaVigna and Malmendier 2004). In this way, our model may help explain why the insurance plans we see in the world appear "too generous" (too low co-pays and deductibles) relative to simulated optimal plans (Cutler and Zeckhauser 2000). Likewise, our model can help explain why physicals and gym memberships may be covered, despite such coverage having little to no insurance value.
} 
subject to zero profit constraint

$$
P=M_{n}(p)(c-p)+\psi(n)
$$

If the representative agent is sophisticated it is again immediate that the equilibrium insurance contract will be efficient. In particular, nudges will be supplied optimally. Matters are different if the agent is naive. In this case, $\hat{m}_{n}$ is independent of $n$, so nudges will only affect $\hat{W}$ insofar as they influence the size of the premium through zero profit constraint (27). Fixing the co-pay, the nudge that maximizes $\hat{W}$ subject to (27) will be the one that minimizes insurer costs. In other words, when the agent is naive, the equilibrium nudge given co-pay $p^{\prime}$ will solve

$$
\min _{n^{\prime}} M_{n^{\prime}}\left(p^{\prime}\right)\left(c-p^{\prime}\right)+\psi\left(n^{\prime}\right)
$$

We thus have:

Proposition 6. If the representative agent is sophisticated then the equilibrium nudge is efficient in the sense that the planner would choose such a nudge. If the representative agent is naive, insurers nudge in a way that minimizes their costs: the equilibrium nudge, $n^{E}$, solves (28) given equilibrium co-pay $p^{E}$.

Note an implication of Proposition 6 when the agent is naive and the equilibrium co-pay is lower than cost $c$ : since the expression being minimized in (28) is decreasing in demand $M_{n}\left(p^{\prime}\right)$, the equilibrium nudge will (weakly) discourage care relative to the default nudge. This is independent of the initial bias: when nudges are available, equilibrium insurance contracts may exacerbate rather than help counteract biases.

Matters are different when insurers' bottom line depends indirectly on whether agents are treated, perhaps because a failure to get treated will lead to greater expenses within the horizon of the insurer. For example, it may be costly to the insurer if an insuree does not get a flu shot. A simple reduced form way to capture this is to extend the model so getting treated may reduce other costs to the insurer, where these other costs are given by $\lambda \chi(M)\left(\lambda \geq 0, \chi(M) \geq 0, \chi^{\prime}(M)<0\right)$. The zero profit constraint is now:

$$
P=M_{n}(p)(c-p)+\psi(n)+\lambda \chi\left(M_{n}(p)\right) .
$$

The limiting case where $\lambda=0$ is the situation we have considered so far - the case where the marginal cost of treatment equals the direct cost of providing treatment (e.g., the cost of a pill). When $\lambda>0$, the marginal cost to the insurer is lower by $\left|\lambda \chi^{\prime}(M)\right|$ because getting treated reduces other expenses. The equilibrium nudge may encourage care in this situation even when agents are naive, as such a nudge may improve the insurer's bottom line. 
Proposition 7. Suppose the zero profit condition is given by Equation (29), the representative agent is naive, and $p^{E}<c$. If $\lambda=0$ then the equilibrium nudge weakly discourages care relative to the default nudge: $M_{n^{E}}\left(p^{E}\right) \leq M_{0}\left(p^{E}\right)$ with strict inequality if $\psi\left(n^{E}\right)>0$. On the other hand, if $\lambda>0$ and the equilibrium co-pay is (exogeneously) restricted to be positive, then the equilibrium nudge may encourage care: it is possible that $M_{n^{E}}\left(p^{E}\right)>M_{0}\left(p^{E}\right)$.

\subsection{Evidence on Private Plans}

These results suggest that competitive forces do not lead to efficient equilibrium insurance contracts when insurees are naive about their biases, but that the insurer will have an incentive to counteract biases when this saves the insurer money. These results may shed light on why more health insurance plans do not incorporate behavioral hazard into their copayment structures. Recall the evidence from Table 2, which summarizes the features of several major insurance plans. Copayments are rarely a function of the health benefit associated with the care for a particular patient. Simply lowering copays for beta blockers and other negative behavioral hazard care could have significant social welfare gains - but the gains may not accrue to the insurer. Beaulieu, Cutler et al. (Commonwealth Report, 2003) estimate that investment in diabetes disease management produces very small monetary gains for insurers over a 10 year horizon, but would produce $\$ 30,000$ per patient in improved quality of life and longevity. ${ }^{44}$

Nevertheless, insurers could increase profits by promoting adherence to medications and treatments that save money over a reasonably short horizon (relative to the typical tenure of their enrollees), and the model suggests that insurers will invest in encouraging care in such instances. For example, flu shots are often given for free and at enrollees' convenience. Perhaps for a similar reason, insurers are also funding research into promoting adherence to certain treatments (Belluck, 2010) and in-house programs aimed at improving adherence, such as Humana's "RxMentor" or United's "Refill and Save" programs. Aetna's tracking suggests that its program targeting chronic disease patients has improved adherence (Sipkoff, 2009). FICO, known for its widely-purchased credit score, is now even selling medication adherence scores to insurers, intended to predict how likely patients are to adhere (NYT; FICO).

\section{Conclusion}

We build a framework for analyzing the relationship between insurance coverage and health care consumption when people make mistakes. The model produces several broad insights about insur-

\footnotetext{
${ }^{44}$ Additionally, while it could be efficient to provide negative prices (subsidies) to use high-value care (Volpp. et. al. 2009), practical considerations may make this infeasible.
} 
ance design and market equilibrium. First, health insurance can provide more than just financial protection: it can lead to more efficient health delivery by counteracting biases that may have led to overconsumption of some services and underconsumption of others. Second, just as the effect of prices on the quantity of care consumed captures the degree of moral hazard in the traditional model, the effect of prices on the health benefit of care can be used to back out a measure of behavioral hazard in this model. This allows us to derive a formula for the optimal co-pay that can be empirically implemented based on these two parameters. Third, unlike in the standard model, government interventions may be welfare-improving over market outcomes even in the absence of selection or externalities. Important next steps include empirically calibrating the optimal copay formula and developing a better understanding of how the government can improve on market outcomes.

The model captures important features of the health care landscape. The areas in which we observe substantial underutilization - such as managment of chronic diseases like diabetes, high blood pressure, asthma, and high cholesterol - are responsible for a substantial share of total health care utilization (Roehrig et al., 2009). Much of the cost of such diseases occurs late in the progression of the condition and likely involves overuse (moral hazard or positive behavioral hazard) following underuse early in the progression of the condition. That many of these domains of care seem sensitive both to small changes in copayments and potentially to behavioral nudges - and that many of the treatments affected seem to be of high health value - suggests that incorporating not only moral hazard but behavioral hazard into our models of optimal insurance design may have large-scale implications for public policy. 


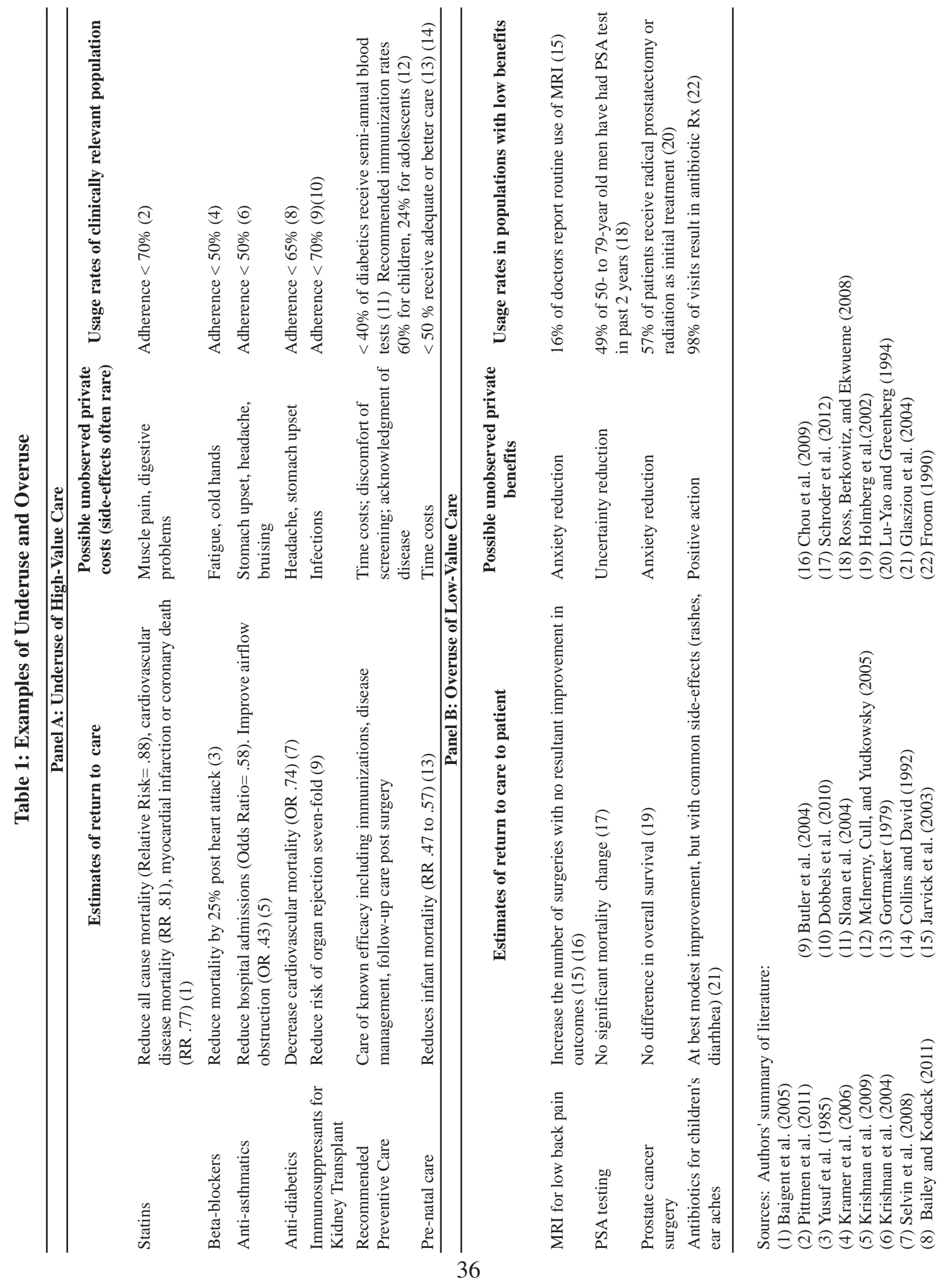




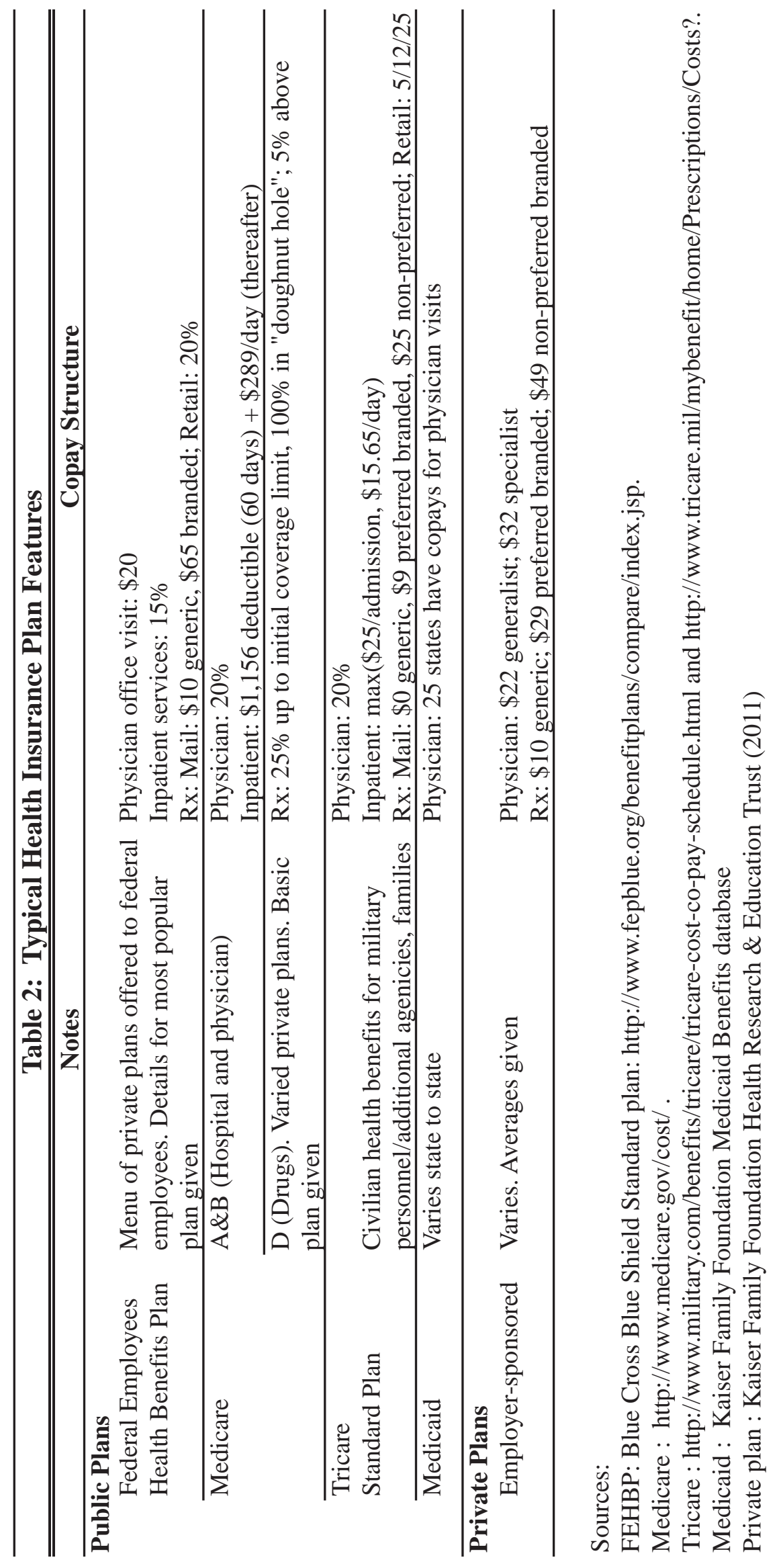




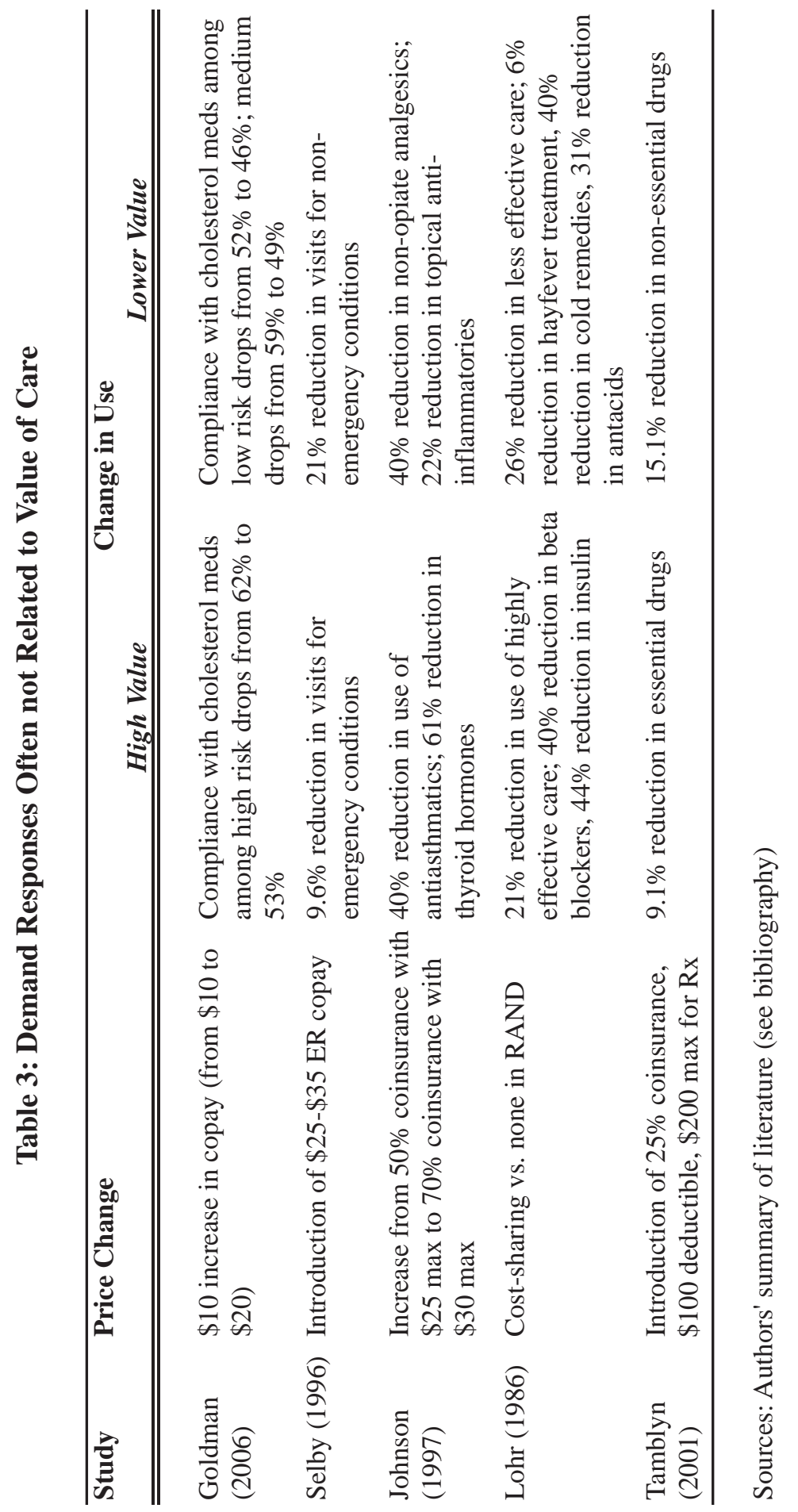


Table 4: Responsiveness to Copays for Different Types of Drugs

\begin{tabular}{lcc|cc|c}
\hline & \multicolumn{2}{c|}{ Baseline } & \multicolumn{2}{c|}{ Change } & \multirow{2}{*}{ Elasticity } \\
& Price & Use & Price & Use & \\
\cline { 2 - 6 } & & & \multirow{2}{*}{0.96} & -0.08 & -0.16 \\
Acute Care & 1.25 & 0.36 & 6.96 & -0.13 & -0.15 \\
Chronic Care & 1.24 & 0.65 & 6.56 & -0.08 & -0.16 \\
Lifestyle & 1.38 & 0.39 & 6.49 &
\end{tabular}

Source: Chandra, Gruber, and McKnight (2010) (unpublished detail)

Notes:

Table shows the responsiveness of drug use by CALPERS HMO enrollees subject to an exogenous price increase.

"Use" is measured in prescriptions per person per month.

Examples of acute care drugs include anticonvulsants, antimalarials, antiangials, vasodilators, and thrombolytics.

Examples of chronic care drugs include analgesics, antivirals, ACE inhibitors, antigout meds, beta-blockers, hypertension drugs, statins, and glaucoma meds.

Examples of lifestyle drugs include acne meds, antihistamines, motion sickness meds, cold remedies, and relief of pain drugs. 


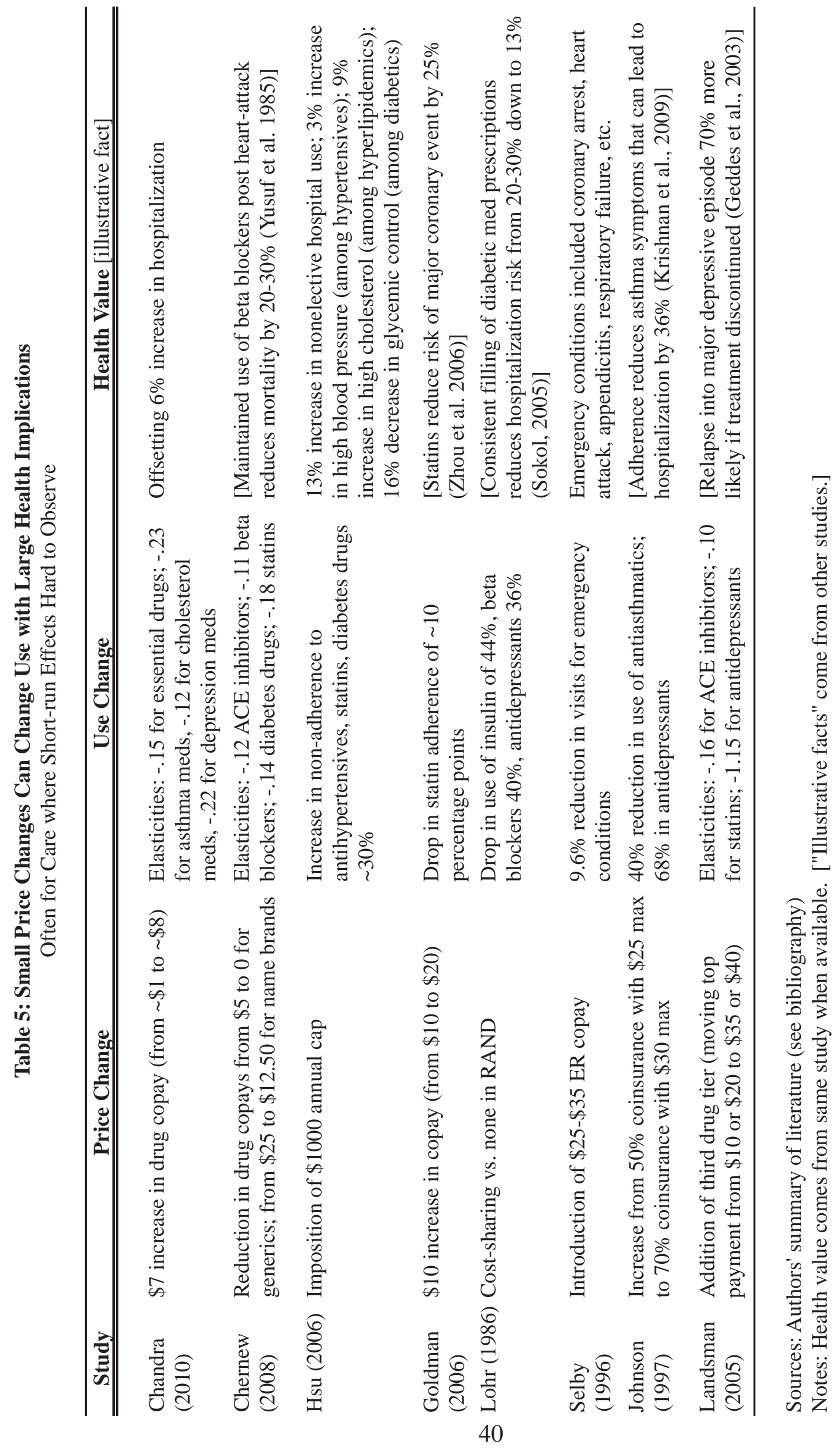




\section{References}

American College of Radiology. (2010). "ACR Appropriateness Criteria." Retrieved February 12, 2010, from http://www.acr.org/SecondaryMainMenuCategories/quality_safety/app_crit eria/pdf/ExpertPanelonNeurologicImaging/LowBackPainDoc7.aspx.

Anderson, K. M., P. M. Odell, et al. (1991). "Cardiovascular disease risk profiles." American Heart Journal 121(1 Pt 2): 293-298.

Andriole, G. L., E. D. Crawford, et al. (2009). "Mortality Results From a Randomized Prostate-Cancer Screening Trial." New England Journal of Medicine 360(13): 1310-1319.

Arrow, K. (1963). "Uncertainty and the Welfare Economics of Medical Care." American Economic Review 53(5): 941-973.

Asch, S. M., E. M. Sloss, et al. (2000). "Measuring Underuse of Necessary Care Among Elderly Medicare Beneficiaries Using Inpatient and Outpatient Claims." JAMA 284(18): 2325-2333.

Baicker, K. and A. Finkelstein (2011). "The Effects of Medicaid Coverage- Learning from the Oregon Experiment." N Engl J Med.

Baigent, C., A. Keech, et al. (2005). "Efficacy and safety of cholesterol-lowering treatment: prospective meta-analysis of data from 90,056 participants in 14 randomised trials of statins." Lancet 366(9493): 1267-1278.

Bailey, C. J. and M. Kodack (2011). "Patient adherence to medication requirements for therapy of type 2 diabetes." International journal of clinical practice 65(3): 314-322.

Bandell, B. (2011). Cover Florida health plan program getting scrapped. South Florida Business Journal.

Beaulieu, N. D., D. Cutler, et al. (2003). "The Business Case for Diabetes Disease Management: A Case Study of HealthPartners and Independent Health Association." The Commonwealth Fund Field Report.

Belluck, P. (2010). For Forgetful, Cash Helps the Medicine Go Down. The New York Times. New York, NY.

Bernheim, B. D. and A. Rangel (2009). "Beyond Revealed Preference: ChoiceTheoretic Foundations for Behavioral Welfare Economics." Quarterly Journal of Economics 124(1): 51-104.

Beshears, J., J. Choi, et al. (2008). "How are preferences revealed?" Journal of Public Economics 92(8): 1787-1794.

Bloche, M. G. (2006). "Consumer-Directed Health Care." New England Journal of Medicine 355(17): 1756-1759.

Butler, J. A., P. Roderick, et al. (2004). "Frequency and Impact of Nonadherence to Immunosuppressants After Renal Transplantation: A Systematic Review." Transplantation 77(5): 769.

Butler, J. A., P. Roderick, et al. (2004). "Frequency and impact of nonadherence to immunosuppressants after renal transplantation: a systematic review." Transplantation 77: 769-776. 
Chandra, A., J. Gruber, et al. (2010). "Patient Cost-Sharing and Hospitalization Offsets in the Elderly." American Economic Review 100(1): 193-213.

Chernew, M. E., A. B. Rosen, et al. (2007). "Value-based insurance design." Health Aff (Millwood) 26(2): w195-203.

Chernew, M. E., M. R. Shah, et al. (2008). "Impact of decreasing copayments on medication adherence within a disease management environment." Health Aff (Millwood) 27(1): 103-112.

Chetty, R. (2009). The Simple Economics of Salience and Taxation, National Bureau of Economic Research.

Chou, R., S. J. Atlas, et al. (2009). "Nonsurgical interventional therapies for low back pain: a review of the evidence for an American Pain Society clinical practice guideline." Spine (Phila Pa 1976) 34(10): 1078-1093.

Chou, R., R. Fu, et al. (2009). "Imaging strategies for low-back pain: systematic review and meta-analysis." Lancet 373(9662): 463-472.

Choudhry, N. K., J. Avorn, et al. (2011). "Full coverage for preventive medications after myocardial infarction." The New England journal of medicine 365(22): 2088-2097.

Cleemput, I., K. Kesteloot, et al. (2002). "A Review of the Literature On the Economics of Noncompliance. Room for Methodological Improvement." Health Policy 59(1): 65-94.

Coco, A., L. Vernacchio, et al. (2010). "Management of Acute Otitis Media After Publication of the 2004 AAP and AAFP Clinical Practice Guideline." Pediatrics 125(2): 214.

Cohen, J. K., R. J. Miller, Jr., et al. (2008). "Ten-year biochemical disease control for patients with prostate cancer treated with cryosurgery as primary therapy." Urology 71(3): 515-518.

Cohen, J. T., P. J. Neumann, et al. (2008). "Does Preventive Care Save Money? Health Economics and the Presidential Candidates." New England Journal of Medicine 358(7): 661-663.

Coker, T. R., L. S. Chan, et al. (2010). "Diagnosis, Microbial Epidemiology, and Antibiotic Treatment of Acute Otitis Media in Children." JAMA: The Journal of the American Medical Association 304(19): 2161.

Collins, J. W., Jr. and R. J. David (1992). "Differences in neonatal mortality by race, income, and prenatal care." Ethnicity \& disease 2(1): 18-26.

Currie, J. and J. Gruber (1996). "Saving Babies: The Efficacy and Cost of Recent Expansions of Medicaid Eligibility for Pregnant Women." Journal of Political Economy 104(6): 1263-1296.

Cutler, D. M., G. Long, et al. (2007). "The value of antihypertensive drugs: a perspective on medical innovation." Health Aff (Millwood) 26(1): 97-110.

Cutler, D. M. and R. J. Zeckhauser (2000). "The Anatomy of Health Insurance." Handbook of health economics 1: 563-643.

DeFrank, J. T., B. K. Rimer, et al. (2009). "Impact of Mailed and Automated Telephone Reminders On Receipt of Repeat Mammograms:: A Randomized Controlled Trial." American journal of preventive medicine 36(6): 459-467.

DellaVigna, S. (2009). "Psychology and Economics: Evidence From the Field." Journal of Economic Literature 47(2): 315-372. 
DellaVigna, S. and U. Malmendier (2004). "Contract Design and Self-Control: Theory and Evidence." The Quarterly Journal of Economics 119(2): 353-402.

Denberg, T. D., T. V. Melhado, et al. (2005). "Predictors of Nonadherence to Screening Colonoscopy." Journal of general internal medicine 20(11): 989995.

Deyo, R. A., S. K. Mirza, et al. (2006). "Back pain prevalence and visit rates: estimates from U.S. national surveys, 2002." Spine (Phila Pa 1976) 31(23): 2724-2727.

Deyo, R. A., J. Rainville, et al. (1992). "What can the history and physical examination tell us about low back pain?" JAMA 268(6): 760-765.

Di Iorio, D., E. Henley, et al. (2000). "A survey of primary care physician practice patterns and adherence to acute low back problem guidelines." Archives of family medicine 9(10): 1015-1021.

Djulbegovic, M., R. J. Beyth, et al. (2010). "Screening for prostate cancer: systematic review and meta-analysis of randomised controlled trials." BMI 341: c4543c4543.

Dobbels, F., T. Ruppar, et al. (2010). "Adherence to the immunosuppressive regimen in pediatric kidney transplant recipients: a systematic review." Pediatric transplantation 14(5): 603-613.

Encinosa, W., D. Bernard, et al. (2010). "Does Prescription Drug Adherence Reduce Hospitalizations and Costs?" NBER Working Paper(15691).

Feldman, R. and B. Dowd (1991). "A New Estimate of the Welfare Loss of Excess Health Insurance." The American Economic Review 81(1): 297-301.

Feldstein, M. (1973). "The Welfare Loss of Excess Health Insurance." Journal of Political Economy 81(2): 251-280.

Finkelstein, A., S. Taubman, et al. (2011). "The Oregon Health Insurance Experiment: Evidence from the First Year." National Bureau of Economic Research Working Paper 17190.

Fojo, T. and C. Grady (2009). "How much is life worth: cetuximab, non-small cell lung cancer, and the $\$ 440$ billion question." Journal of the National Cancer Institute 101(15): 1044-1048.

Froom, J., L. Culpepper, et al. (1990). "Diagnosis and antibiotic treatment of acute otitis media: report from International Primary Care Network." BMI 300(6724): 582-586.

Gann, C. (2011) "Man Dies From Toothache, Couldn't Afford Meds." ABC News.

Gao, X., D. Nau, et al. (2000). "The Relationship of Disease Severity, Health Beliefs and Medication Adherence Among HIV Patients." AIDS care 12(4): 387-398.

Geddes, J. R., S. M. Carney, et al. (2003). "Relapse prevention with antidepressant drug treatment in depressive disorders: a systematic review." Lancet 361: 653-661.

Gertler, P. and T. Simcoe (2009). "Disease Management: Helping Patients (Who Don't) Help Themselves."

Gibson, T., X. Song, et al. (2010). "Cost Sharing, Adherence, and Health Outcomes in Patients with Diabetes." The American journal of managed care 16(8): 589.

Gibson, T. B., S. Wang, et al. (2011). "A Value-Based Insurance Design Program at a Large Company Boosted Medication Adherence for Employees with Chronic Illnesses." Health affairs 30(1): 109-117. 
Glasziou, P. P., C. B. Del Mar, et al. (2004). "Antibiotics for acute otitis media in children." Cochrane database of systematic reviews(1): CD000219.

Glazer, J. and T. G. McGuire (2012). "A Welfare Measure of "Offset Effects" in Health Insurance." Journal of Public Economics 96(5-6): 520-523.

Goldman, D. and T. J. Philipson (2007). "Integrated Insurance Design in the Presence of Multiple Medical Technologies." American Economic Review 97(2): 427432.

Goldman, D. P. and G. F. Joyce (2012). Consumer Demand and Health Effects of Cost-Sharing. Oxford Handbook of the Economics of the Biopharmaceutical Industry. USA, Oxford University Press: 365.

Goldman, D. P., G. F. Joyce, et al. (2004). "Pharmacy benefits and the use of drugs by the chronically ill." Jama 291(19): 2344-2350.

Goldman, D. P., G. F. Joyce, et al. (2006). "Varying Pharmacy Benefits with Clinical Status: The Case of Cholesterol-Lowering Therapy." American Journal of Managed Care 12(1): 21-28.

Gortmaker, S. L. (1979). "The effects of prenatal care upon the health of the newborn." American journal of public health 69(7): 653-660.

Griffin, K. J. and T. D. Elikin (2001). "Non-Adherence in Pediatric Transplantation: A Review of the Existing Literature." Pediatric Transplantation 5(4): 246249.

Grijalva, C. G., J. P. Nuorti, et al. (2009). "Antibiotic Prescription Rates for Acute Respiratory Tract Infections in US Ambulatory Settings." JAMA: The Journal of the American Medical Association 302(7): 758.

Gruber, J. (2008). "Covering the Uninsured in the U.S." NBER Working Paper 13758.

Gruber, j. and B. Koszegi (2001). "Is Addiction "Rational"? Theory and Evidence." Quarterly Journal of Economics 115(4): 1261.

Haas, G. P., N. Delongchamps, et al. (2008). "The worldwide epidemiology of prostate cancer: perspectives from autopsy studies." The Canadian Journal of Urology 15(1): 3866-3871.

Haynes, R. B., E. Ackloo, et al. (2008). "Interventions for Enhancing Medication Adherence." Cochrane database syst Rev 2.

Health Resources and Services Administration (2011). Prenatal - First Timester Care Access, Department of Health and Human Services.

Hodgson, T. A. and L. Cai (2001). "Medical care expenditures for hypertension, its complications, and its comorbidities." Medical care 39(6): 599-615.

Holmberg, L., A. Bill-Axelson, et al. (2002). "A randomized trial comparing radical prostatectomy with watchful waiting in early prostate cancer." The New England journal of medicine 347(11): 781-789.

Howlader, N., A. Noone, et al. (2011). SEER Cancer Statistics Review, 1975-2008. Bethesda, MD, National Cancer Institute.

Hsu, J. T., M. Price, et al. (2006). "Unintended Consequences of Caps on Medicare Drug Benefits." New England Journal of Medicine 354(22): 2349-2359.

Hyman, D. J. and V. N. Pavlik (2001). "Characteristics of Patients with Uncontrolled Hypertension in the United States." New England Journal of Medicine 345(7): 479-486. 
Jarvik, J. G., W. Hollingworth, et al. (2003). "Rapid magnetic resonance imaging vs radiographs for patients with low back pain: a randomized controlled trial." JAMA 289(21): 2810-2818.

Jarvik, J. G., W. Hollingworth, et al. (2003). "Rapid Magnetic Resonance Imaging Vs Radiographs for Patients with Low Back Pain." JAMA: the journal of the American Medical Association 289(21): 2810.

Jencks, S. F., E. D. Huff, et al. (2003). "Change in the quality of care delivered to Medicare beneficiaries, 1998-1999 to 2000-2001." JAMA 289(3): 305-312.

Johnson, R. E., M. J. Goodman, et al. (1997). "The impact of increasing patient prescription drug cost sharing on therapeutic classes of drugs received and on the health status of elderly HMO members." Health services research 32(1): 103-122.

Kahneman, D. and P. P. Wakker (1997). "Back to Bentham? Explorations of Experienced Utility." Quarterly Journal of Economics.

Kaiser Family Foundation. (2012). "State Health Facts." 2012, from http://www.statehealthfacts.org/profileglance.jsp?rgn=39.

Kaiser Family Foundation and Health Research and Education Trust (2011). Employer Health Benefits 2011 Survey. Menlo Park, CA.

Koszegi, B. (2003). "Health anxiety and patient behavior." Journal of Health Economics 22(6): 1073-1084.

Kramer, J. M., B. Hammill, et al. (2006). "National evaluation of adherence to betablocker therapy for 1 year after acute myocardial infarction in patients with commercial health insurance." American heart journal 152(3): 454 e451458.

Krishnan, J. A., S. Q. Davis, et al. (2009). "An umbrella review: corticosteroid therapy for adults with acute asthma." The American journal of medicine 122(11): 977-991.

Krishnan, J. A., K. A. Riekert, et al. (2004). "Corticosteroid Use After Hospital Discharge Among High-Risk Adults with Asthma." American journal of respiratory and critical care medicine 170(12): 1281-1285.

Krishnan, J. A., K. A. Riekert, et al. (2004). "Corticosteroid use after hospital discharge among high-risk adults with asthma." American journal of respiratory and critical care medicine 170: 1281-1285.

Laibson, D. (1997). "Golden Eggs and Hyperbolic Discounting." The Quarterly Journal of Economics 112(2): 443.

Landsman, P. B., W. Yu, et al. (2005). "Impact of 3-tier pharmacy benefit design and increased consumer cost-sharing on drug utilization." The American journal of managed care 11(10): 621-628.

Landsman, P. B., W. Yu, et al. (2005). "Impact of 3-tier pharmacy benefit design and increased consumer cost-sharing on drug utilization." Am J Manag Care 11(10): 621-628.

Leonhardt, D. (2010). Health Care's Uneven Road to a New Era. The New York TImes. New York, NY.

Levy, H. and D. Meltzer (2008). "The Impact of Health Insurance On Health." Annu. Rev. Public Health 29: 399-409. 
Liebman, J. and R. Zeckhauser (2008). Simple Humans, Complex Insurance, Subtle Subsidies, National Bureau of Economic Research.

Lohr, K. N., R. H. Brook, et al. (1986). "Use of Medical Care in the Rand Health Insurance Experiment: Diagnosis-and Service-Specific Analyses in a Randomized Controlled Trial." Medical care 24(9): 1-87.

Lu-Yao, G. L. and E. R. Greenberg (1994). "Changes in prostate cancer incidence and treatment in USA." Lancet 343(8892): 251-254.

Luo, X., R. Pietrobon, et al. (2004). "Estimates and patterns of direct health care expenditures among individuals with back pain in the United States." Spine (Phila Pa 1976) 29(1): 79-86.

Macfarlane, J., W. Holmes, et al. (1997). "Influence of Patients' Expectations On Antibiotic Management of Acute Lower Respiratory Tract Illness in General Practice: Questionnaire Study." Bmj 315(7117): 1211-1214.

Manning, W. G., J. P. Newhouse, et al. (1987). "Health Insurance and the Demand for Medical Care: Evidence from a Randomized Experiment." American Economic Review 77(3): 251-277.

McCaig, L. F., R. E. Besser, et al. (2002). "Trends in Antimicrobial Prescribing Rates for Children and Adolescents." JAMA: The Journal of the American Medical Association 287(23): 3096.

McCombs, J. S., M. B. Nichol, et al. (1994). "The Costs of Interrupting Antihypertensive Drug Therapy in a Medicaid Population." Medical care: 214-226.

McCormick, D. P., T. Chonmaitree, et al. (2005). "Nonsevere Acute Otitis Media: A Clinical Trial Comparing Outcomes of Watchful Waiting Versus Immediate Antibiotic Treatment." Pediatrics 115(6): 1455-1465.

McGlynn, E. A., S. M. Asch, et al. (2003). "The Quality of Health Care Delivered to Adults in the United States." New England Journal of Medicine 348(26): 2635-2645.

McInerny, T. K., W. L. Cull, et al. (2005). "Physician reimbursement levels and adherence to American Academy of Pediatrics well-visit and immunization recommendations." Pediatrics 115(4): 833-838.

McKenney, J., W. Munroe, et al. (1992). "Impact of an Electronic Medication Compliance Aid On Long-Term Blood Pressure Control." The Journal of Clinical Pharmacology 32(3): 277.

Miller, K., M. Wang, et al. (2007). "Paclitaxel Plus Bevacizumab Versus Paclitaxel Alone for Metastatic Breast Cancer." New England Journal of Medicine 357(26): 2666-2676.

Mullainathan, S., J. Schwartzstein, et al. (2012). "A Reduced-Form Approach to Behavioral Public Finance." Annual Review of Economics Forthcoming.

National Center for Chronic Disease Prevention and Health Promotion (2011). Vital Signs: High Blood Pressure and Cholesterol. C. f. D. Control.

National Committee for Quality Assurance (2005). "The state of health care quality." Technical Report.

Ness, R. M., A. M. Holmes, et al. (2000). "Cost-Utility of One-Time Colonoscopic Screening for Colorectal Cancer at Various Ages." The American journal of gastroenterology 95(7): 1800-1811. 
Newhouse, J. P. (2006). "Reconsidering the Moral Hazard-Risk Avoidance Tradeoff." Journal of Health Economics 25(5): 1005-1014.

Newhouse, J. P. and The Insurance Experiment Group (1993). Free for all? Lessons from the RAND Health Insurance Experiment. A RAND Study. Cambridge and London, Harvard University Press.

Nyman, J. A. (1999). "The Economics of Moral Hazard Revisited." Journal of Health Economics 18(6): 811-824.

O'donoghue, T. and M. Rabin (1999). "Doing It Now or Later." American Economic Review: 103-124.

Osterberg, L. and T. Blaschke (2005). "Adherence to Medication." New England Journal of Medicine 353(5): 487-497.

Parker-Pope, T. (2011). Keeping Score on How You Take Your Medicine. The New York Times. New York, NY.

Parkington, S. and et al. (2009). "Barriers to Breast Cancer Screening in a Managed Care Population." Managed Care(April).

Pauly, M. V. and F. E. Blavin (2008). "Moral Hazard in Insurance, Value-Based Cost Sharing, and the Benefits of Blissful Ignorance." Journal of Health Economics 27(6): 1407-1417.

Pear, R. (2011). Program offering waivers for health law is ending. New York Times.

Perlroth, D. J., D. P. Goldman, et al. (2010). "The potential impact of comparative effectiveness research on U.S. health care expenditures." Demography 47 Suppl: S173-190.

Pittman, D. G., W. Chen, et al. (2011). "Adherence to statins, subsequent healthcare costs, and cardiovascular hospitalizations." The American journal of cardiology 107(11): 1662-1666.

Psaty, B. M., T. D. Koepsell, et al. (1990). "The relative risk of incident coronary heart disease associated with recently stopping the use of beta-blockers." JAMA: the journal of the American Medical Association 263(12): 1653-1657.

Quincy, L. (2009). "State policies to encourage high-deductible and limited-benefit health plans: costs, consituents and concerns." Mathematica Policy Brief.

Ribeiro, R. A., P. K. Ziegelmann, et al. (2011). "Impact of statin dose on major cardiovascular events: A mixed treatment comparison meta-analysis involving more than 175,000 patients." International journal of cardiology.

Roehrig, C., G. Miller, et al. (2009). "National Health Spending by Medical Condition, 1996--2005." Health affairs 28(2): w358-w367.

Ross, L. E., Z. Berkowitz, et al. (2008). "Use of the prostate-specific antigen test among U.S. men: findings from the 2005 National Health Interview Survey." Cancer Epidemiology, Biomarkers \& Prevention 17: 636-644.

Rowe, J. W., T. Brown-Stevenson, et al. (2008). "The Effect of Consumer-Directed Health Plans On the Use of Preventive and Chronic Illness Services." Health affairs 27(1): 113-120.

Rubin, R. R. (2005). "Adherence to Pharmacologic Therapy in Patients with Type 2 Diabetes Mellitus." The American journal of medicine 118(5): 27-34.

Schedlbauer, A., P. Davies, et al. (2010). "Interventions to Improve Adherence to Lipid Lowering Medication." Cochrane Database Syst Rev 3(3). 
Schroder, F., J. Hugosson, et al. (2012). "Prostate-cancer mortality at 11 years of follow-up." New England Journal of Medicine 366: 981-990.

Schroeder, F. H. and others (2009). "Screening and Prostate-Cancer Mortality in a Randomized European Study." New England Journal of Medicine 360(13): 1320-1328.

Schroeder, K., T. Fahey, et al. (2004). "How Can We Improve Adherence to Blood Pressure-Lowering Medication in Ambulatory Care?: Systematic Review of Randomized Controlled Trials." Archives of internal medicine 164(7): 722.

Schroeder, K., T. Fahey, et al. (2008). "Interventions for Improving Adherence to Treatment in Patients with High Blod Pressure in Ambulatory Settings." Cochrane Library 2008(4).

Selby, J. V., B. H. Fireman, et al. (1996). "Effect of a Copayment On Use of the Emergency Department in a Health Maintenance Organization." New England Journal of Medicine 334(10): 635-642.

Selby, J. V., B. H. Fireman, et al. (1996). "Effect of a copayment on use of the emergency department in a health maintenance organization." $\mathrm{N}$ Engl J Med 334(10): 635-658.

Selvin, E., S. Bolen, et al. (2008). "Cardiovascular outcomes in trials of oral diabetes medications: a systematic review." Archives of internal medicine 168(19): 2070-2080.

Senst, B. L., L. E. Achusim, et al. (2001). "Practical approach to determining costs and frequency of adverse drug events in a health care network." American journal of health-system pharmacy : AJHP : official journal of the American Society of Health-System Pharmacists 58(12): 1126-1132.

Sheehan, N. (2010). "Magnetic resonance imaging for low back pain: indications and limitations." Ann Rheum Dis 69(1): 7-11.

Sipkoff, M. (2009). Improved adherence highlights specialty pharmacy's potential. Managed Care Magazine.

Sloan, F. A., M. A. Bethel, et al. (2004). "Adherence to guidelines and its effects on hospitalizations with complications of type 2 diabetes." The review of diabetic studies : RDS 1(1): 29-38.

Sokol, M. C., K. A. McGuigan, et al. (2005). "Impact of medication adherence on hospitalization risk and healthcare cost." Med Care 43(6): 521-530.

Solanki, G., H. H. Schauffler, et al. (2000). "The direct and indirect effects of costsharing on the use of preventive services." Health Services Research 34(6): 1331-1350.

Soni, A. (2008). "Ear infections (otitis media) in children (0-17): use and expenditures, 2006." MEPS Statistical Brief \#228 228.

Soumerai, S. B., T. J. McLaughlin, et al. (1997). "Adverse outcomes of underuse of beta-blockers in elderly survivors of acute myocardial infarction." JAMA 277: 115-121.

Spiro, D. M., K. Y. Tay, et al. (2006). "Wait-and-See Prescription for the Treatment of Acute Otitis Media." JAMA: The Journal of the American Medical Association 296(10): 1235.

Steinbrook, R. (2008). "Saying no isn't NICE: The travails of Britain's National Institute for Health and Clinical Excellence." N Engl J Med 359: 1977-1981. 
Strandbygaard, U., S. F. Thomsen, et al. (2010). "A Daily SMS Reminder Increases Adherence to Asthma Treatment: A Three-Month Follow-Up Study." Respiratory medicine 104(2): 166-171.

Strine, T. W. and J. M. Hootman (2007). "US national prevalence and correlates of low back and neck pain among adults." Arthritis Rheum 57(4): 656-665.

Tamblyn, R., R. Laprise, et al. (2001). "Adverse events associated with prescription drug cost-sharing among poor and elderly persons." JAMA 285(4): 421-429.

Tamblyn, R., R. Laprise, et al. (2001). "Adverse events associated with prescription drug cost-sharing among poor and elderly persons." JAMA : the journal of the American Medical Association 285(4): 421-429.

Thaler, R. and C. Sunstein (2008). Nudge: Improving Decisions About Health, Wealth, and Happiness, Yale University Press.

Van Dulmen, S., E. Sluijs, et al. (2007). "Patient Adherence to Medical Treatment: A Review of Reviews." BMC Health Services Research 7(1): 55.

Volpp, K. G., M. V. Pauly, et al. (2009). "P4P4P: An Agenda for Research On Pay-forPerformance for Patients." Health affairs 28(1): 206-214.

Wang, G. (2011). "Cost-Effectiveness of Hypertension Control." iHEA Abstract.

World Health Organization, T. (2003). Adherence to Long-Term Therapies: Evidence for Action. Geneva, WHO.

Yusuf, S., R. Peto, et al. (1985). "Beta blockade during and after myocardial infarction: an overview of the randomized trials." Progress in cardiovascular diseases 27(5): 335-371.

Yusuf, S., R. Peto, et al. (1985). "Beta blockade during and after myocardial infarction: an overview of the randomized trials." Progress in Cardiovascular Diseases 27: 335-371.

Zeckhauser, R. (1970). "Medical Insurance: A Case Study of the Tradeoff Between Risk Spreading and Appropriate Incentives." Journal of Economic Theory 2(1): 10-26.

Zhou, Z., E. Rahme, et al. (2006). "Are Statins Created Equal? Evidence From Randomized Trials of Pravastatin, Simvastatin, and Atorvastatin for Cardiovascular Disease Prevention." American Heart Journal 151(2): 273281.

Zhou, Z., E. Rahme, et al. (2006). "Are statins created equal? Evidence from randomized trials of pravastatin, simvastatin, and atorvastatin for cardiovascular disease prevention." American Heart Journal 151: 273-281. 


\section{Appendix}

\section{A Proofs}

Proof of Propositions 1 and 2. Proposition 1 is just a special case of 2, so we will prove Proposition 2. Welfare given co-pay $p$ is given by

$W(p)=(1-q) U(y-P(p))+q\left\{\int_{\underline{s}}^{s(p)} U(y-P(p)-s) d F(s)+\int_{s(p)}^{\bar{s}} U(y-P(p)-s+b(s)-p) d F(s)\right\}$

where

$$
P(p)=M(p)(c-p)
$$

and

$$
s(p)= \begin{cases}\underline{s} & \text { if } p<b(\underline{s})+\varepsilon(\underline{s}) \\ \text { the value } s^{\prime} \text { satisfying } b\left(s^{\prime}\right)+\varepsilon\left(s^{\prime}\right)=p & \text { if } b(\underline{s})+\varepsilon(\underline{s}) \leq p \leq b(\bar{s})+\varepsilon(\bar{s}) \\ \bar{s} & \text { if } p>b(\bar{s})+\varepsilon(\bar{s}) .\end{cases}
$$

We will proceed by first showing that there exist finite bounds $\underline{p}, \bar{p}$ with $\underline{p}<\bar{p}$ such that if an optimal value of $p$ exists, there is one that lies in $[\underline{p}, \bar{p}]$. Since $W(p)$ is continuous in $p$ and $[\underline{p}, \bar{p}]$ is a compact set, this will imply that an optimum exists.

We have $W^{\prime}(p)=0$ for $p>b(\bar{s})+\varepsilon(\bar{s})$. Thus, if there is an optimum there is one that satisfies $p \leq b(\bar{s})+\varepsilon(\bar{s})$. In addition, we have

$$
W^{\prime}(p)=q\left(E\left[U^{\prime}(C)\right]-E\left[U^{\prime}(C) \mid m=1\right]\right)
$$

for $p<b(\underline{s})+\varepsilon(\underline{s})$. When $p$ is sufficiently low, $E\left[U^{\prime}(C)\right] \geq E\left[U^{\prime}(C) \mid m=1\right]$ and $W^{\prime}(p) \geq 0 .{ }^{45}$ As a result, there exists some threshold $\underline{p}$ such that if an optimum exists there is one that satisfies $p \geq \underline{p}$, and we have established the existence of an optimum.

\footnotetext{
${ }^{45}$ To see this, note that for $p<b(\underline{s})+\varepsilon(\underline{s}), E\left[U^{\prime}(C)\right] \geq E\left[U^{\prime}(C) \mid m=1\right]$ iff$$
U^{\prime}(y-P) \geq \int_{\underline{s}}^{\bar{s}} U^{\prime}(y-P-s+b(s)-p) d F(s) .
$$

Since $b(s)-s$ is decreasing in $s$, the right hand side of (32) is less than or equal to $U^{\prime}(y-P-\bar{s}+b(\bar{s})-p)$ by concavity of $U$. Noting further that $U^{\prime}(y-P-\bar{s}+b(\bar{s})-p) \leq U^{\prime}(y-P)$ for $p<b(\bar{s})-\bar{s}$, (32) holds for such $p$.
} 
If demand is positive and price sensitive at an optimal value of $p$, then $d W / d p=0$ at this value. Equivalently, (10) must equal 0 at such a value. Finally, re-arranging (10) for the case where $M(p)>0, M^{\prime}(p)<0$, and $p \neq 0$ yields (11).

Proof of Corollary 1. Let

$$
M_{W}^{S}(p) \equiv-M^{\prime}(p)(c-p)-I \cdot M(p)
$$

equal the normalized welfare impact of a marginal increase in the co-pay (by Equation (7)). It is clear that $p^{S}=c$ when $U^{\prime \prime}=0$. When $U^{\prime \prime}<0$, we have $M_{W}^{S}(p) \leq 0$ for $p \geq c$, with strict inequality at $p=c$ (since $I \cdot M(c)>0$ ). Hence, $p^{S}<c$ when $U^{\prime \prime}<0$, establishing the first part of the Corollary.

To establish the second part, we have $M_{W}^{S}(p) \geq 0$ for $p \leq p_{F I}$ with strict inequality at $p=p_{F I}$ if $M^{\prime}\left(p_{F I}\right)<0$ (since $p_{F I}<c$ and $I=0$ at $p_{F I}$ ). Hence, $p^{S} \geq p_{F I}$ with strict inequality so long as $M^{\prime}\left(p_{F I}\right)<0$.

Proof of Corollary 2. Let

$$
M_{W}^{B}(p) \equiv-M^{\prime}(p)\left(c-p+\varepsilon^{\prime}\right)-I \cdot M(p)
$$

equal the normalized welfare impact of a marginal increase in the co-pay (by Equation (10)).

We will prove each part of the corollary by example. Let $b(s)=s$ and $\varepsilon(s)=\tilde{\varepsilon}$ for all $s$, where $\tilde{\varepsilon}$ equals some real number. For the first part of the corollary, suppose $\tilde{\varepsilon}>c-\underline{s}$. In this case, for all $p \leq c$ we have $M(p)=q$, for $p \in[0, c]$ we have $I \cdot M(p) \geq 0$ with equality only at $p=0$, and for $p<0$ we have $I \cdot M(p)<0$. As a result, for $p \in[0, c]$, we have $M_{W}^{B}(p) \leq 0$ with equality only at $p=0$, and for $p<0$ we have $M_{W}^{B}(p)>0$. Hence, $p=0$ is a local maximum, and the optimal co-pay, $p^{B}$, is either 0 or some value greater than $c$. A sufficient condition for the optimal co-pay to be above $c$ is then for welfare when the co-pay equals 0 to be lower than welfare when the co-pay is high enough that nobody demands treatment, or for $W(0)=U(y-q c)<(1-q) U(y)+q E[U(y-s) \mid s \geq \underline{s}]=W(\infty)$, which holds, for example, if $U(y-q c)<E[U(y-s) \mid s \geq \underline{s}]$.

For the second part, let $\tilde{\varepsilon}=-\bar{s}$ and $-\varepsilon^{\prime}>c$, where $\varepsilon^{\prime}$ is evaluated at $p=0$. In this case, $M(p)=M^{\prime}(p)=0$ for all $p>0$, so $M_{W}^{B}(p)=0$ for all $p>0$. Moreover, $M(0)=0$, while $-M_{-}^{\prime}(0)\left(c+\varepsilon^{\prime}\right)<0$, implying that $M_{W-}^{B}(0)<0$ and hence $p^{B}<0$. 
Proof of Proposition 3. Expand

$$
H(p)=E[m(p) b-s]=q\left(\int_{\underline{s}}^{s(p)}-s d F(s)+\int_{s(p)}^{\bar{s}} b(s)-s d F(s)\right)
$$

so

$$
\begin{aligned}
H^{\prime}(p) & =(b(s(p))-s(p)) M^{\prime}(p)+s(p) M^{\prime}(p) \\
& =b(s(p)) M^{\prime}(p)
\end{aligned}
$$

which implies that

$$
\frac{H^{\prime}(p)}{M^{\prime}(p)}=b(s(p))
$$

By definition,

$$
b(s(p))+\varepsilon(s(p))=p .
$$

Using (35) to substitute for $b(s(p))$ in Equation (36) and re-arranging yields

$$
\varepsilon(s(p))=p-\frac{H^{\prime}(p)}{M^{\prime}(p)} .
$$

Proof of Proposition 4. (Part 1) By definition,

$$
b\left(s_{0}\right)+\varepsilon_{0}\left(s_{0}\right)=p_{0} .
$$

Since $M_{n}\left(p_{n}\right) \geq M_{0}\left(p_{0}\right)$, we also have that

$$
b\left(s_{0}\right)+\varepsilon_{n}\left(s_{0}\right) \geq p_{n} .
$$

Inserting the first equality into the second expression yields

$$
p_{n}-p_{0} \leq \varepsilon_{n}\left(s_{0}\right)-\varepsilon_{0}\left(s_{0}\right) \leq-\varepsilon_{0}\left(s_{0}\right),
$$

where the final inequality follows from the assumption that $\varepsilon_{n}\left(s_{0}\right) \leq 0$.

The proof of the second part is similar, and hence omitted. 
Proof of Proposition 5. Perceived welfare given co-pay $p$ is given by

$\hat{W}(p)=(1-q) U(y-P(p))+q\left\{\int_{\underline{s}}^{\hat{s}(p)} U(y-P(p)-s) d F(s)+\int_{\hat{s}(p)}^{\bar{s}} U(y-P(p)-s+b(s)-p) d F(s)\right\}$

where

$$
P(p)=M(p)(c-p)
$$

and

$$
\hat{s}(p)= \begin{cases}\underline{s} & \text { if } p<b(\underline{s})+\hat{\varepsilon}(\underline{s}) \\ \text { the value } s^{\prime} \text { satisfying } b\left(s^{\prime}\right)+\hat{\varepsilon}\left(s^{\prime}\right)=p & \text { if } b(\underline{s})+\hat{\varepsilon}(\underline{s}) \leq p \leq b(\bar{s})+\hat{\varepsilon}(\bar{s}) \\ \bar{s} & \text { if } p>b(\bar{s})+\hat{\varepsilon}(\bar{s}) .\end{cases}
$$

We will establish existence of an equilibrium co-pay using the same technique we used to establish the existence of an optimal co-pay in proving Proposition 2: we show that there exist finite bounds $\underline{p}, \bar{p}$ with $\underline{p}<\bar{p}$ such that if an equilibrium value of $p$ exists, there is one that lies in $[\underline{p}, \bar{p}]$. Since $\hat{W}(p)$ is continuous in $p$ and $[\underline{p}, \bar{p}]$ is a compact set, this will imply that an equilibrium value of $p$ exists.

We have $\hat{W}^{\prime}(p)=0$ for $p>\max \{b(\bar{s})+\hat{\varepsilon}(\bar{s}), b(\bar{s})+\varepsilon(\bar{s})\}$. Thus, if there is an equilibrium value of $p$, there is one that satisfies $p \leq \max \{b(\bar{s})+\hat{\varepsilon}(\bar{s}), b(\bar{s})+\varepsilon(\bar{s})\}$. In addition, we have

$$
\hat{W}^{\prime}(p)=q\left(E\left[U^{\prime}(C)\right]-E\left[U^{\prime}(C) \mid m=1\right]\right)
$$

for $p<\min \{b(\underline{s})+\hat{\varepsilon}(\underline{s}), b(\underline{s})+\varepsilon(\underline{s})\}$. As established in the proof of Propositions 1 and 2, $E\left[U^{\prime}(C)\right] \geq E\left[U^{\prime}(C) \mid m=1\right]$ when $p$ is sufficiently low, so $\hat{W}^{\prime}(p) \geq 0$ when $p$ is sufficiently low. As a result, there exists some threshold $\underline{p}$ such that if an equilibrium value of $p$ exists there is one that satisfies $p \geq \underline{p}$, and we have established the existence of an equilibrium value of $p$.

If demand and forecasted demand are positive and price-sensitive at an equilibrium value of $p$, then $\hat{W}^{\prime}(p)=0$ at this value. Equivalently, (22) must equal 0 at such a value. Re-arranging (22) for the case where we further have that $p^{E} \neq 0$ yields (23).

The fact that welfare is weakly higher under $p^{B}$ than under $p^{E}$ is immediate from the fact that $p^{E}$ solves the planner's problem.

Proof of Proposition 6. Proof in text. 
Proof of Proposition 7. Generally, the equilibrium nudge solves

$$
\min _{n^{\prime}} M_{n^{\prime}}\left(p^{E}\right)\left(c-p^{E}\right)+\psi\left(n^{\prime}\right)+\lambda \chi\left(M_{n^{\prime}}\left(p^{E}\right)\right) .
$$

Take the case where $\lambda=0$ and $p^{E}<c$. In this case, we must have

$$
M_{n^{E}}\left(p^{E}\right)\left(c-p^{E}\right)+\psi\left(n^{E}\right) \leq M_{0}\left(p^{E}\right)\left(c-p^{E}\right)
$$

since $\psi(0)=0$. For this inequality to hold, we must have $M_{n^{E}}\left(p^{E}\right) \leq M_{0}\left(p^{E}\right)$ with strict inequality if $\psi\left(n^{E}\right)>0$.

Now suppose $\lambda>0$ and $p^{E} \in[0, c)$. Then we may have $M_{n^{E}}\left(p^{E}\right)>M_{0}\left(p^{E}\right)$. To see this, suppose there are two nudges, $n=0,1$, with $\psi(0)=\psi(1)=0$ and $M_{1}(p)>M_{0}(p)$ for all $p \in[0, c)$. Further, suppose $\chi(M)=1-M$ and $\lambda>c$. For all $p \in[0, c)$, we then have that

$$
\begin{aligned}
M_{1}(p)(c-p)+\psi(1)+\lambda \chi\left(M_{1}(p)\right) & =M_{1}(p)(c-p-\lambda)+\lambda \\
& <M_{0}(p)(c-p-\lambda)+\lambda \\
& =M_{0}(p)(c-p)+\psi(0)+\lambda \chi\left(M_{0}(p)\right),
\end{aligned}
$$

so $n^{E}=1$.

\section{B Extensions}

\section{B.1 Heterogeneity in Diseases and Treatments}

Suppose the social planner is restricted to setting the same co-pay for a range of treatments, for example because of high administrative costs. In practice, many insurance policies set the same copay across a category of treatments (e.g., a drug formulary tier). How does this restriction change the logic behind how the social planner should design insurance?

We can extend the model to allow for multiple diseases, where there are a finite number of such diseases and each is associated with a single treatment. For simplicity, suppose that each treatment costs society $c$ and is associated with the same co-pay $p$. Let the probability of disease $j$ be denoted by $q_{j}$. The agent cannot have multiple diseases at once. The probability that the agent is healthy is now $1-\sum_{j} q_{j}$.

Continue to define $m(p)$ as the individual's demand for care at a given price: when the individual has disease $j, m(p)$ equals 1 if and only if $b_{j}(s)>p$ for the rational agent and $b_{j}(s)+\varepsilon_{j}(s)>p$ 
for the behavioral agent. The social planner's problem is now to maximize

$$
W=\left(1-\sum_{j} q_{j}\right) U(y-P)+\sum_{j} q_{j} E_{j}[U(y-P-s+m(b-p))]
$$

subject to break-even constraint

$$
P=M(p)(c-p)
$$

where $M(p) \equiv \sum_{j} q_{j} E_{j}[m(p)]$ equals aggregate demand for medical care given co-pay $p$, and $E_{j}$ represents the expectation operator conditional on the individual having disease $j$. Let $M_{j}(p)=$ $q_{j} E_{j}[m(p)]$ equal total demand for treating disease $j$, and $m_{j}(p)=E_{j}[m(p)]$ equal demand conditional on disease $j$.

As before, raising the co-pay diminishes insurance value and may also affect people's choice of care. The analysis of rational actors proceeds almost identically to the single disease case. Raising the co-pay from $p$ to $p^{\prime}$ leads all those whose true benefit of treatment falls between $p$ and $p^{\prime}$ to no longer take care. Adding multiple diseases and treatments does not make it more difficult to calculate the welfare impact of reducing utilization: independent of the treatment, the fact that someone is marginal reveals that his value of the treatment lies between $p$ and $p^{\prime}$.

Consider a price $p$ such that, for each disease $j$, total demand for treatment is positive $M_{j}(p)>$ 0 and price sensitive $M_{j}^{\prime}(p)<0$. Then, totally differentiating $W$ with respect to $p$ and converting into a money metric yields:

$$
\frac{d W}{d p} / \frac{d W}{d y}=-M^{\prime}(p)(c-p)-I \cdot M(p)
$$

where $I=\left(\frac{E\left[U^{\prime}(C) \mid m=1\right]-E\left[U^{\prime}(C)\right]}{E\left[U^{\prime}(C)\right]}\right)$ again equals the insurance value to consumers. Equation (45) is exactly the same as (7): introducing multiple diseases does not affect the logic behind insurance design when people are rational. In particular, $-M^{\prime}(p)(c-p)$ continues to represent the welfare gain from reducing moral hazard and $M^{\prime}(p)$ remains a sufficient statistic for measuring this gain. In the rational model, it is unnecessary to consider whether raising the co-pay affects demand for statins or antibiotics to measure the welfare impact: independent of the treatment, anyone on the margin has the same treatment value.

Matters are more interesting when individuals are behavioral. Recall that when the co-pay is raised from $p$ to $p^{\prime}$ all those whose decision value of some treatment falls between $p$ and $p^{\prime}$ no longer take care. Since the wedge between decision and hedonic valuations may depend on the treatment, understanding which treatments are forgone may provide information about the welfare effects of reducing utilization. 
Totally differentiating $W$ with respect to $p$ and converting into a money metric yields:

$$
\frac{d W}{d p} / \frac{d W}{d y}=-M^{\prime}(p)(c-p)-E\left[m_{i}^{\prime}(p) \varepsilon_{i}^{\prime}\right]-I \cdot M(p)
$$

where $\varepsilon_{i}^{\prime}=\frac{U\left(y-P-s_{i}\right)-U\left(y-P-s_{i}-\varepsilon_{i}\right)}{E\left[U^{\prime}(C)\right]}$ is an increasing function of $\varepsilon_{i}$ (this expression should be evaluated at the disease severity of the marginal agent given disease state $i, s_{i}$ ). This equation illustrates the discussion above. The third term again represents the reduction in insurance value for all treated individuals. The first two terms, $-M^{\prime}(p)(c-p)-E\left[m_{i}^{\prime}(p) \varepsilon_{i}^{\prime}\right]$, now capture the welfare effect of lower utilization: the standard term, $-M^{\prime}(p)(c-p)$, is corrected by $-E\left[m_{i}^{\prime}(p) \varepsilon_{i}^{\prime}\right]$, reflecting that certain treatment decisions are not made according to true value (the expectation is taken over disease states). When raising the co-pay reduces demand for diseases associated with positive behavioral hazard $\left(\varepsilon_{i}^{\prime}>0\right)$ by a substantial amount more than it reduces demand for diseases associated with negative behavioral hazard $\left(\varepsilon_{i}^{\prime}<0\right)$ then this term is positive and raising the co-pay has the benefit of decreasing overutilization. In the opposite scenario, raising the co-pay can have the cost of reducing underutilization. Looking at the overall demand response can be misleading: to understand the welfare impact of raising the co-pay, it is useful to understand the extent to which people make errors when making certain treatment decisions (e.g., whether to take statins, antibiotics), as well as the demand response treatment-by-treatment (elasticity of demand for statins and antibiotics separately).

\section{B.1.1 Empirical Implementation}

How can we estimate the welfare impact of raising the co-pay and calibrate the optimal co-pay formula when raising the co-pay affects demand for multiple treatments? A key result is that the lessons from Section 5.1 carry over to this setting: we can continue to look at overall health and demand responses to higher co-pays. This fact is a direct consequence of the following result.

Proposition 8. Consider a co-pay, $p$, at which demand is price sensitive, so $M^{\prime}(p)<0$. Let $s_{j}(p)$ denote the disease severity of the marginal agent with disease $j$ given the co-pay if one exists (otherwise, set $s_{j}(p)=\bar{s}$ ) and let $\varepsilon_{j}(p) \equiv \varepsilon_{j}\left(s_{j}(p)\right)$ denote the behavioral error of the marginal agent. The following relationship holds between the marginal behavioral error, demand responses, and health responses:

$$
\frac{E\left[m_{i}^{\prime}(p) \varepsilon_{i}(p)\right]}{M^{\prime}(p)}=p-\frac{H^{\prime}(p)}{M^{\prime}(p)} .
$$

Proof. Let $h_{i}(p)=E_{i}\left[m_{i}(p) b_{i}(s)-s\right]$. It is clear that $h_{i}^{\prime}(p)=m_{i}^{\prime}(p) b_{i}\left(s_{i}(p)\right)$, so $H^{\prime}(p)=$ $E\left[m_{i}^{\prime}(p) b_{i}\left(s_{i}(p)\right)\right]=E\left[m_{i}^{\prime}(p)\left(p-\varepsilon_{i}\left(s_{i}(p)\right)\right)\right]=p M^{\prime}(p)-E\left[m_{i}^{\prime}(p) \varepsilon_{i}\left(s_{i}(p)\right)\right]$. Rearranging this equality yields (47). 
Proposition 8 implies that we can use the same data to estimate the model whether or not there is heterogeneity. Plugging Equation (47) into (46) (after approximating $\varepsilon_{i}^{\prime} \approx \varepsilon_{i}$ ) gives exactly Equation (13); i.e., Equation (13) continues to be a valid approximation of the welfare impact of raising the co-pay. This implies that to estimate the welfare impact of raising the co-pay across a broad category of treatments and to calibrate the optimal co-pay formula, it is enough to look at aggregate demand and health responses.

\section{B.2 Heterogeneity in Biases}

In the baseline single disease/treatment model, biases are homogeneous and expressed with certainty - i.e., $\varepsilon(s)$ is non-stochastic (fixing $s$ ). However, biases may be heterogeneous across the population, for example if some people are more present-biased or inattentive than others. This can be modeled by introducing a finite number of behavioral types, $\theta \in \Theta$, where a given type $\theta^{\prime}$ has measure $\alpha_{\theta^{\prime}} \in[0,1]$ in the population and is characterized by making decisions according to error map $\varepsilon_{\theta^{\prime}}(s)$; that is, type $\theta^{\prime}$ demands treatment when sick if and only if $b(s)+\varepsilon_{\theta^{\prime}}(s)>p$.

The analysis of this case proceeds identically to that of the multiple disease model of Subsection B.1. To see this, note that the extension allowing for heterogeneous biases is formally identical to a special case of the extension with multiple diseases, where $\varepsilon_{j}(s)$ varies in $j$, but $b_{j}(s)$ does not. For this reason, the welfare impact of raising the co-pay can be expressed as $\frac{d W}{d p} / \frac{d W}{d y}=-M^{\prime}(p)(c-p)-E\left[m_{\theta}^{\prime}(p) \varepsilon_{\theta}^{\prime}\right]-I \cdot M(p)$, and can be approximated by Equation (13).

\section{B.3 Forgetfulness}

\section{B.3.1 Optimal Insurance}

As a final extension, consider the psychology of "forgetfulness", where somebody might completely forget to get treated or fill a prescription (or in general fail to follow through on intended courses of treatment). Allowing for such forgetfulness does not change the logic of how to set copays because it does not affect who is marginal. To see this, extend the basic one disease/one treatment model so that, with some exogeneous probability $\phi$ independent of severity, the individual will forget to get treated if sick, and, with probability $1-\phi$, will remember and tradeoff decision benefits with costs (i.e., will compare $b(s)+\varepsilon(s)$ to $p$ ). In this case, welfare can be written as

$$
\begin{aligned}
W=(1-q) U(y-P) & +q \phi E[U(y-P-s) \mid \text { sick }] \\
& +q(1-\phi) E[U(y-P-s+m(b-p) \mid \text { sick, remember }]
\end{aligned}
$$


subject to

$$
P=\underbrace{q(1-\phi) E[m(p) \mid \text { sick, remember }]}_{=M(p)}(c-p) .
$$

Differentiating $W$ with respect to $p$ and normalizing then gives exactly (10): allowing for forgetfulness does not change how copays affect welfare. ${ }^{46}$

Note also that the model with forgetfulness is formally equivalent to a special case of the extensions analyzed in Subsections B.1 and B.2 (where $\varepsilon=-\infty$ with probability $\phi$ ), so the results derived in those subsections also hold here. In particular, allowing for forgetfulness does not alter how we can estimate the welfare impact of raising the co-pay; Equation (13) continues to be a valid approximation.

\section{B.3.2 Market Provision}

Incorporating forgetfulness into the market equilibrium model, suppose that individuals forecast that they will be forgetful with probability $\hat{\phi}$, where $\hat{\phi}=\phi$ for a sophisticate and $\hat{\phi}=0$ for a naif. In equilibrium, the market will supply insurance plan $(P, p)$ to maximize the agent's perceived expected utility

$$
\begin{aligned}
\hat{W}=(1-q) U(y-P) & +q \hat{\phi} E[U(y-P-s) \mid \text { sick }] \\
& +q(1-\hat{\phi}) E[U(y-P-s+\hat{m}(b-p)) \mid \text { sick, remember }]
\end{aligned}
$$

subject to zero profit constraint

$$
P=M(p)(c-p)
$$

Introducing forgetfulness does not change the equilibrium co-pay formula: the equilibrium copay is still implicitly given by (23). However, adding forgetfulness illustrates that the nature of the bias is important in determining whether profit motives will lead insurers to set co-pays that deviate from cost in the optimal direction. To see this, again consider the simple case of a riskneutral naive agent (so $I=\hat{I}=0, \varepsilon^{\prime}=\varepsilon$, and $\hat{\varepsilon}=\hat{\varepsilon}^{\prime}=\hat{\phi}=0$ ). In this case, the optimal co-pay formula reduces to

$$
p^{B}=c+\varepsilon\left(s\left(p^{B}\right)\right)
$$

The intuition behind why the optimal co-pay is independent of the level of forgetfulness is that

\footnotetext{
${ }^{46}$ Of course, the degree of forgetfulness will change values of the parameters in the formula, e.g., the higher $\phi$, the lower $M(p)$.
} 
this level does not affect the marginal social value of treatment. Consequently, the co-pay that maximizes health net of costs, $\mathcal{E}(\cdot)$, is independent of the forgetting probability $\phi$.

On the other hand, the equilibrium co-pay in this case satisfies

$$
p^{E}=c+\frac{M\left(p^{E}\right)-\hat{M}\left(p^{E}\right)}{\left|\dot{M}\left(p^{E}\right)\right|}=c+\frac{(1-\phi) M^{R}\left(p^{E}\right)-\hat{M}\left(p^{E}\right)}{(1-\phi)\left|\dot{M}^{R}\left(p^{E}\right)\right|},
$$

where $M^{R}(p)=\frac{1}{1-\phi} M(p)$ equals demand conditional on the agent remembering to consider treatment. The equilibrium co-pay thus depends on the level of forgetfulness since this level influences the degree to which the agent misforecasts her demand for treatment. In the special case where forgetfulness is the only bias $(\varepsilon=0),(52)$ and (53) give us that $p^{B}=c$ and $p^{E}=c-\frac{\phi}{1-\phi} \frac{M^{R}\left(p^{E}\right)}{\left|\dot{M}^{R}\left(p^{E}\right)\right|}:^{47}$ while the optimal co-pay equals cost, the equilibrium co-pay is below cost. ${ }^{48}$

\section{Case Study on Hypertension}

High blood pressure is a prevalent and potentially deadly condition: 68 million adults in the U.S. have high blood pressure (CDC Vital Signs 2011), which is associated with adverse events such as heart attacks and stroke that carry with them serious health consequences including risk of death. The cost of treating hypertension and its consequences is high: Hodgson and Cai (2001) estimate the expenditures associated with hypertension and its effects to be about $12.5 \%$ of health expenditures, or over $\$ 5,000$ annually per hypertensive patient $(\$ 3,800$ in 1998 , inflated using $\mathrm{CPI}$ ). We use this important disease as a stylized example to illustrate implications of a model that incorporates behavioral hazard, examining: (1) the existence of effective treatments to avoid adverse consequences; (2) adherence and responsiveness to nudges; (3) effects of adherence on blood pressure and health outcomes.

The Efficacy of Drug Treatments and Consequences of Uncontrolled Hypertension: There are several classes of drugs (such as beta blockers and ACE inhibitors) that aim to reduce patients' blood pressure and thereby reduce the chance of serious downstream health events. The medical literature suggests that policies that increased compliance with anti-hypertensives would have substantial health effects through lowering blood pressure. Use of beta-blockers post-heart attack can reduce subsequent mortality by more than $40 \%$ (Soumerai, 1997). Hsu et al. (2006) found a $30 \%$ drop in anti-hypertensive compliance was associated with a $3 \%$ increase in hypertension and

\footnotetext{
${ }^{47}$ Note that when forgetfulness is the only bias, $M^{R}(p)=\hat{M}(p)$.

${ }^{48}$ More starkly (but perhaps unrealistically), consider a treatment that is overvalued at the margin $(\varepsilon(s)>0$ for all $s)$, but which the agent may forget to consider $(\phi>0)$. While the optimal co-pay in this case lies above cost, the equilibrium co-pay will be below cost for sufficiently large $\phi$.
} 
subsequent hospital and emergency department use. Anderson et al. (1991) present a model of how risk factors such as hypertension are associated with adverse events including heart attack and stroke in a non-elderly population, and calculate an overall risk of mortality from cardiovascular disease. For a 50 year old non-smoking, non-diabetic man with somewhat elevated cholesterol, a decrease in systolic blood pressure from $160 \mathrm{mmHg}$ to $140 \mathrm{mmHg}$ would reduce the risk of 10 -year cardiovascular disease mortality by 2.5 percentage points; a decrease in diastolic blood pressure from 100 to 90 would reduce it by 5 percentage points. Long, Cutler et al. (2006) find that the advent of anti-hypertensive drugs reduced blood pressure in the population over age 40 by 10 percent or more, and averted 86,000 deaths from cardiovascular disease in 2001 and 833,000 hospitalizations for stroke and heart attack. They also note that in addition to these health improvements observed under existing use, under "guideline" use these numbers might have been twice as high - suggesting that improved patient adherence (as well as physician prescribing practices) might generate substantial health gains.

Adherence and Responsiveness to Nudges and Copays: Patient adherence to anti-hypertensives is not only far from perfect, but is sensitive to both finances and nudges. Schroeder et al. (2008) review the evidence on adherence to anti-hypertensives and efforts to improve it based on nonfinancial strategies, including studies that use reminder systems and innovative packaging. Overall adherence to hypertensive therapies is around $50 \%-70 \%$, but such interventions were able to increase it by around 10-20 percentage points. Several of the studies they review that focus on strategies such as reminders find not only improvements in adherence but reductions in blood pressure. McKenney (1992) for example finds that an intervention including electronic medicine caps and reminders increased adherence by 20 percentage points and reduced systolic and diastolic blood pressure by $10-20 \mathrm{mmHg}$. Several of the studies examining price-elasticity of pharmaceutical use mentioned above look at results for anti-hypertensives in particular. Many of these estimates cluster around -0.1 to -0.2 (including Chandra, Gruber and McKnight 2010; Landsman et al. 2005; and Chernew et al. 2008). ${ }^{49}$ The drop in use found by Hsu et al. (2006) was in response to the imposition of a cap on drug benefits (with the imposition of caps in general resulting in a decrease of $30 \%$ in pharmaceutical use but an offsetting increase in non-elective hospital use of $13 \%$ and emergency department use of $9 \%$ ).

\footnotetext{
${ }^{49}$ Chandra et al. (2010) find an elasticity of -0.09 for anti-hypertensives among chronically ill HMO patients, based on an increase in copayments of around $\$ 5$ (unpublished detail). Chernew et al. (2008) estimate elasticities of -.12 for ACE inhibitors/ARBs and -. 11 for beta blockers, based on copay reductions from $\$ 5$ to 0 for generics and $\$ 25$ to $\$ 12.50$ for branded drugs. Landsman et al. (2005) find elasticities of -.16 for ARBs and CCBs and -.14 for ACE inhibitors, based on the introduction of tiering that raised copays by $\$ 20$ or less. Lohr et al. (1986) find a reduction in use of beta blockers of $40 \%$ among those exposed to copayments in the RAND HIE versus those who were not, but the finding was not statistically significant. Similarly, Hsu et al. (2006) find that the imposition of a cap on Medicare beneficiaries' drug benefits results in an increase in anti-hypertensive non-adherence of $30 \%$.
} 
Effects on Health Outcomes: Unlike the Choudhry study examined in the main text, most of these studies do not individually include the full set of outcomes needed to perform welfare calculations under models of moral hazard and behavioral hazard (and indeed, many do not even report the change in total (all-payer) drug expenditures), but they can be used in combination to illustrate the point. For example, the drop in total drug utilization in one of the policy experiments studied in Chandra et al. is around $\$ 23$ a month when copays go up to about $\$ 7.50$ per drug among members who use about 1.4 drugs per month, or to a coinsurance rate of about $22 \%$ of the $\$ 48$ per month in drug spending. Following the logic from the Choudhry example in the main text, the standard moral hazard model would thus suggest that this copay increase generated less than about $\$ 5$ per month in health cost (with the remaining \$18 reduction in utilization attributable to reduced moral hazard) - which is clearly at odds with the observed changes in health outcomes. ${ }^{50}$ The imposition of benefit caps studied by Hsu et al. produced a similar magnitude decline in utilization, and was associated with an increase in death rates of 0.7 percentage points, along with increased blood pressure, cholesterol, and blood sugar - implying a cost from increased mortality alone of $\$ 7,000$ using conventional valuations. ${ }^{51}$

Another way of synthesizing this stylized evidence is to calculate the likely mortality reduction from changes in copayments or nudges that promote adherence and compare it to the cost of the intervention. Estimates suggest that interventions like a $\$ 5$ reduction in copayments or a nudge with comparable demand response applied to the $40 \%$ of people who are not adherent could increase compliance with anti-hypertensive therapy by something like 10 percentage points, resulting in a decrease in blood pressure of $15 \mathrm{~mm}$ and subsequent 3 percentage point reduction in deaths from cardiovascular disease (and even greater gains for readily-identifiable subpopulations like those with previous heart attacks, who may see a 40 percent reduction in subsequent mortality). This reduction in mortality would be valued at $\$ 30,000$. This might be compared to the cost of an adherence program. If such a program worked on $1 / 4$ of the people to whom it was applied and came at a cost of less than $\$ 7,500$, then it would be welfare improving based on this outcome alone. Given the estimates of the total annual cost of treating hypertensive patients (around \$5,000), and that these interventions largely operate on the low-marginal-cost margin of increased adherence to prescriptions that have already been obtained through an office visit, this seems likely to be the case

\footnotetext{
${ }^{50}$ Chandra et al do not measure health directly, but the increased hospitalization rate is indicative of substantially worse outcomes. These stylized examples ignore the fact that the price of drugs is not likely an adequate proxy for their actual marginal cost.

${ }^{51}$ How we value reductions in mortality and improvements in health is of course a subject of major debate. Some estimates of the monetary value of life-years are derived from revealed preference arguments that are inherently grounded in a rational agent model, but others are based on labor market earnings, social welfare arguments, etc. Commonly used estimates cluster around \$100,000 per “quality-adjusted life year" and \$1 million per death averted (although this clearly varies based on the age at which death is averted and the life expectancy gained - averting the death of a young health worker might be valued at $\$ 5$ million).
} 
- and is consistent with meta-analysis suggesting that interventions to manage use of hypertension medications were highly cost-effective (Wang 2011).

As the Cutler et al. (2007) estimates suggest, there could be around 90,000 total deaths averted through greater compliance with optimal antihypertensive therapy, suggesting the scope of such gains could be vast. Furthermore, this can be viewed as a lower bound, insofar as there are many other health benefits of reduced hypertension beyond just the deaths from cardiovascular disease - including improved quality of life and lower incidence of expensive hospitalizations, etc. This example clearly over-simplifies (to the point of inaccuracy) complex medical pathways, but is meant to be illustrative both about the steps involved in such a calculation and the rough order of magnitude of the potential effects. 